\title{
Trinidad and Tobago: Statistical Appendix
}

The Statistical Appendix for Trinidad and Tobago was prepared by a staff team of the International Monetary Fund as background documentation for the periodic consultation with the member country. It is based on the information available at the time it was completed on September 28, 2006. The views expressed in this document are those of the staff team and do not necessarily reflect the views of the government of Trinidad and Tobago or the Executive Board of the IMF.

The policy of publication of staff reports and other documents by the IMF allows for the deletion of market-sensitive information.

To assist the IMF in evaluating the publication policy, reader comments are invited and may be sent by e-mail to publicationpolicy@imf.org.

Copies of this report are available to the public from

International Monetary Fund $\bullet$ Publication Services

700 19th Street, N.W. • Washington, D.C. 20431

Telephone: (202) 6237430 • Telefax: (202) 6237201

E-mail: publications@imf.org • Internet: http://www.imf.org

Price: $\$ 18.00$ a copy

\section{International Monetary Fund Washington, D.C.}





\title{
INTERNATIONAL MONETARY FUND
}

\section{TRINIDAD AND TOBAGO}

\begin{abstract}
Statistical Appendix
Prepared by a staff team consisting of Irineu de Carvalho Filho, Christopher Faircloth (WHD) and Zsofia Arvai (MFD)
\end{abstract}

Approved by the Western Hemisphere Department

September 28, 2006

Contents

Page

Real Sector

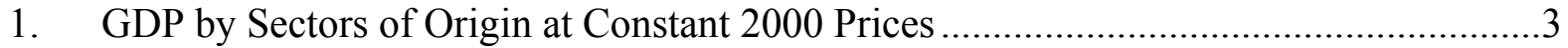

2. GDP by Sectors of Origin, Current Market Prices ......................................................4

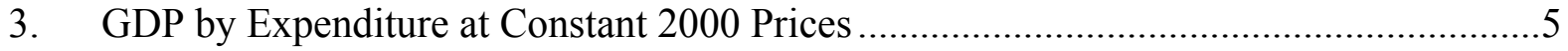

4. GDP by Final Expenditure at Current Market Prices ....................................................6

5. Savings and Investment at Current Market Prices ................................................. 7

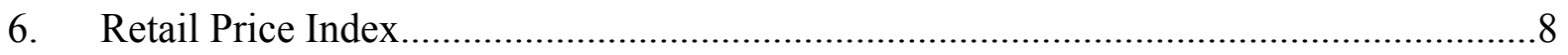

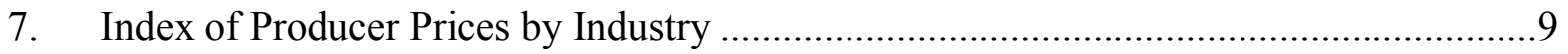

8. Labor Force and Employment ..................................................................... 10

9. Growth of Production, Earnings, Employment, Hours and Costs in Manufacturing .....11

Fiscal Sector

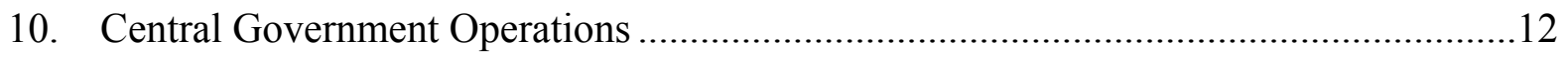

11. Central Government Revenue and Grants .......................................................13

12. Ratios of Central Government Revenue and Grants..................................................14

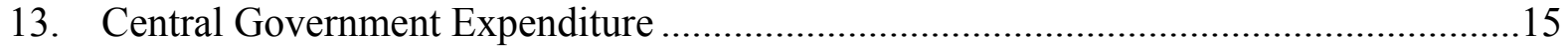

14. Central Government Expenditure Ratios ................................................................... 16

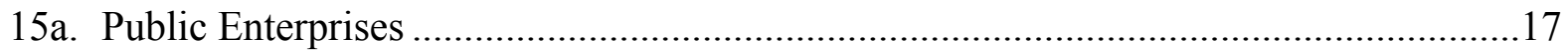

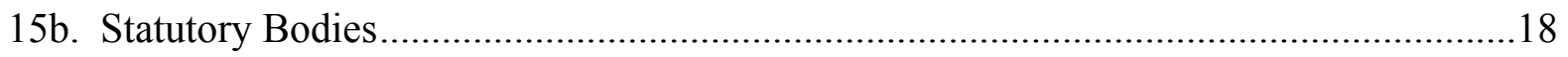

16. Consolidated Nonfinancial Public Sector ............................................................. 19

17. Summary Accounts of the Consolidated Financial System .......................................20 
Monetary Sector

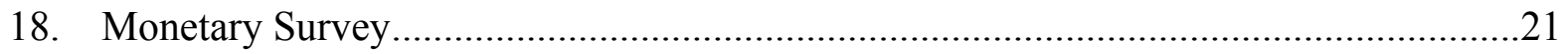

19. Summary Accounts of the Central Bank .................................................................22

20. Consolidated Accounts of the Commercial Banks ..................................................23

21. Commercial Bank Loans and Advances ...............................................................24

22. Summary Accounts of the Nonbank Financial Institutions (NBFIs) ...........................25

23. Financial Soundness Indicators - Banking Sector, 2000-2005 ...............................26

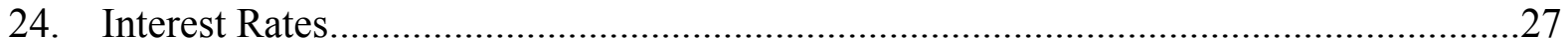

External Sector

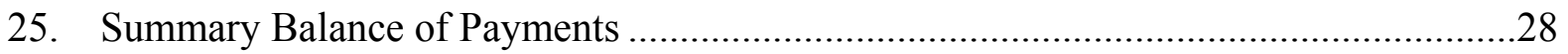

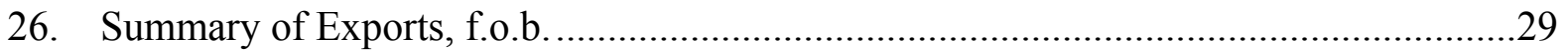

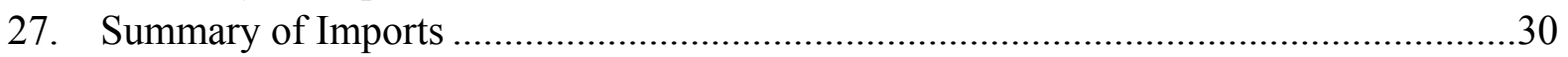

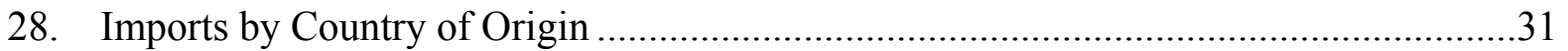

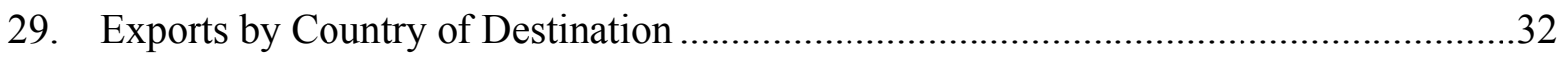

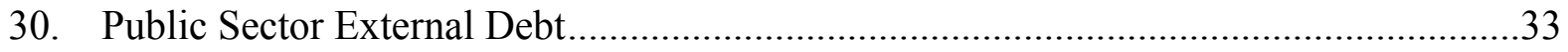


Table 1. Trinidad and Tobago: GDP by Sectors of Origin at Constant 2000 Prices

\begin{tabular}{|c|c|c|c|c|c|}
\hline & 2001 & 2002 & 2003 & 2004 & 2005 \\
\hline Real GDP (2000 prices) $1 /$ & 53,512 & 57,747 & 65,777 & 71,743 & 77,427 \\
\hline Energy sector & 16,971 & 19,260 & 25,285 & 27,420 & 29,677 \\
\hline Crude oil and gas production & 9,573 & 11,003 & 14,364 & 15,551 & 17,246 \\
\hline Refining, including LNG & 2,775 & 3,468 & 5,932 & 6,419 & 6,532 \\
\hline Service, marketing, and asphalt & 2,293 & 2,170 & 2,252 & 2,404 & 2,261 \\
\hline Petrochemicals & 2,329 & 2,619 & 2,737 & 3,046 & 3,638 \\
\hline Nonenergy sector & 36,542 & 38,488 & 40,493 & 44,322 & 47,750 \\
\hline Agriculture & 758 & 824 & 698 & 532 & 572 \\
\hline Export & 10 & 18 & 13 & 13 & 10 \\
\hline Domestic & 407 & 404 & 402 & 369 & 431 \\
\hline Sugar & 341 & 401 & 283 & 150 & 131 \\
\hline Manufacturing & 3,980 & 4,129 & 4,329 & 4,805 & 5,439 \\
\hline Construction & 4,227 & 4,012 & 4,950 & 5,571 & 6,442 \\
\hline Distribution & 8,169 & 8,277 & 8,442 & 8,715 & 9,287 \\
\hline Hotel & 238 & 246 & 238 & 277 & 272 \\
\hline Government & 3,830 & 3,970 & 3,931 & 3,955 & 4,032 \\
\hline Financial services & 7,362 & 8,211 & 8,814 & 10,728 & 11,786 \\
\hline Other & 7,927 & 8,577 & 9,134 & 9,295 & 9,889 \\
\hline Less: imputed service charge & $-2,004$ & $-1,990$ & $-2,181$ & $-2,442$ & $-2,521$ \\
\hline Value added tax $2 /$ & 2,056 & 2,233 & 2,138 & 2,886 & 2,553 \\
\hline
\end{tabular}

Source: Central Statistical Office.

1/ Sectoral constant prices GDP data for 2001 onwards is calculated using different methodology from previous years. 
Table 2. Trinidad and Tobago: GDP by Sectors of Origin, Current Market Prices

\begin{tabular}{|c|c|c|c|c|c|}
\hline & 2001 & 2002 & 2003 & 2004 & 2005 \\
\hline & \multicolumn{5}{|c|}{ (In millions of Trinidad and Tobago dollars) } \\
\hline Nominal GDP & 59,239 & 60,665 & 75,681 & 85,209 & 102,128 \\
\hline Energy sector & 15,559 & 14,765 & 25,605 & 29,842 & 40,616 \\
\hline Crude oil and gas production & 8,686 & 7,951 & 14,889 & 18,261 & 23,923 \\
\hline Refining, including LNG & 2,399 & 2,557 & 4,608 & 4,329 & 6,313 \\
\hline Service, marketing, and asphalt & 2,329 & 2,535 & 2,864 & 3,449 & 4,407 \\
\hline Petrochemicals & 2,145 & 1,722 & 3,244 & 3,804 & 5,974 \\
\hline Nonenergy sector & 43,680 & 45,900 & 50,076 & 55,367 & 61,512 \\
\hline Agriculture & 708 & 787 & 675 & 729 & 741 \\
\hline Export & 11 & 19 & 14 & 16 & 14 \\
\hline Domestic & 413 & 469 & 427 & 472 & 479 \\
\hline Sugar & 284 & 298 & 234 & 242 & 248 \\
\hline Manufacturing & 4,074 & 4,494 & 4,477 & 5,032 & 5,690 \\
\hline Construction & 4,353 & 4,092 & 5,197 & 6,184 & 7,731 \\
\hline Distribution & 8,724 & 9,287 & 9,902 & 10,624 & 12,212 \\
\hline Hotel & 236 & 255 & 265 & 310 & 357 \\
\hline Government & 4,714 & 4,333 & 5,560 & 5,964 & 6,523 \\
\hline Financial services & 7,506 & 8,890 & 9,182 & 10,539 & 11,709 \\
\hline Other & 9,070 & 9,173 & 9,962 & 9,925 & 10,412 \\
\hline Less: imputed service charge & 2,116 & 2,187 & 2,493 & 2,889 & 3,190 \\
\hline \multirow[t]{2}{*}{ Plus: VAT } & 2,179 & 2,401 & 2,364 & 3,171 & 2,948 \\
\hline & \multicolumn{5}{|c|}{ (In percent of GDP) } \\
\hline Petroleum sector & 26.3 & 24.3 & 33.8 & 35.0 & 39.8 \\
\hline Crude oil & 14.7 & 13.1 & 19.7 & 21.4 & 23.4 \\
\hline Refining & 4.1 & 4.2 & 6.1 & 5.1 & 6.2 \\
\hline Service, marketing, and asphalt & 3.9 & 4.2 & 3.8 & 4.0 & 4.3 \\
\hline Petrochemicals & 3.6 & 2.8 & 4.3 & 4.5 & 5.8 \\
\hline Nonpetroleum sector & 73.7 & 75.7 & 66.2 & 65.0 & 60.2 \\
\hline Agriculture & 1.2 & 1.3 & 0.9 & 0.9 & 0.7 \\
\hline Export & 0.0 & 0.0 & 0.0 & 0.0 & 0.0 \\
\hline Domestic & 0.7 & 0.8 & 0.6 & 0.6 & 0.5 \\
\hline Sugar & 0.5 & 0.5 & 0.3 & 0.3 & 0.2 \\
\hline Manufacturing & 6.9 & 7.4 & 5.9 & 5.9 & 5.6 \\
\hline Construction & 7.3 & 6.7 & 6.9 & 7.3 & 7.6 \\
\hline Distribution & 14.7 & 15.3 & 13.1 & 12.5 & 12.0 \\
\hline Hotel & 0.4 & 0.4 & 0.4 & 0.4 & 0.3 \\
\hline Government & 8.0 & 7.1 & 7.3 & 7.0 & 6.4 \\
\hline Financial services & 12.7 & 14.7 & 12.1 & 12.4 & 11.5 \\
\hline Other & 15.3 & 15.1 & 13.2 & 11.6 & 10.2 \\
\hline Less: imputed service charge & 3.6 & 3.6 & 3.3 & 3.4 & 3.1 \\
\hline Plus: VAT & 3.7 & 4.0 & 3.1 & 3.7 & 2.9 \\
\hline
\end{tabular}

Source: Central Statistical Office. 
Table 3. Trinidad and Tobago: GDP by Expenditure at Constant 2000 Prices

\begin{tabular}{|c|c|c|c|c|c|}
\hline & 2001 & 2002 & 2003 & 2004 & 2005 \\
\hline & \multicolumn{5}{|c|}{ (In millions of Trinidad and Tobago dollars) } \\
\hline Domestic expenditure & 48,565 & 55,974 & 60,395 & 64,861 & 69,179 \\
\hline Consumption & 38,818 & 46,102 & 50,259 & 53,415 & 57,203 \\
\hline Private sector & 32,270 & 37,035 & 40,738 & 42,775 & 44,660 \\
\hline Government & 6,548 & 9,067 & 9,521 & 10,640 & 12,543 \\
\hline Gross capital formation & 9,747 & 9,872 & 10,136 & 11,446 & 11,975 \\
\hline Net exports & 4,947 & 1,773 & 5,383 & 6,881 & 8,248 \\
\hline Exports of goods and nonfactor services & 30,295 & 25,437 & 26,526 & 28,525 & 34,288 \\
\hline Imports of goods and nonfactor services & 25,348 & 23,665 & 21,143 & 21,644 & 26,040 \\
\hline \multirow[t]{2}{*}{ GDP at constant prices } & 53,512 & 57,747 & 65,777 & 71,743 & 77,427 \\
\hline & \multicolumn{5}{|c|}{ (Annual percentage changes) } \\
\hline Domestic expenditure & 9.8 & 15.3 & 7.9 & 7.4 & 6.7 \\
\hline Consumption & 9.4 & 18.8 & 9.0 & 6.3 & 7.1 \\
\hline Private sector & 9.9 & 14.8 & 10.0 & 5.0 & 4.4 \\
\hline Government & 6.6 & 38.5 & 5.0 & 11.8 & 17.9 \\
\hline Gross capital formation & 11.5 & 1.3 & 2.7 & 12.9 & 4.6 \\
\hline Net exports & -30.7 & -64.2 & 203.6 & 27.8 & 19.9 \\
\hline Exports of goods and nonfactor services & -0.4 & -16.0 & 4.3 & 7.5 & 20.2 \\
\hline Imports of goods and nonfactor services & 8.8 & -6.6 & -10.7 & 2.4 & 20.3 \\
\hline \multirow[t]{2}{*}{ GDP at constant prices } & 4.2 & 7.9 & 13.9 & 9.1 & 7.9 \\
\hline & \multicolumn{5}{|c|}{ (Contribution to growth) } \\
\hline Domestic expenditure & 8.4 & 13.8 & 7.7 & 6.8 & 6.0 \\
\hline Net exports & -4.3 & -5.9 & 6.3 & 2.3 & 1.9 \\
\hline GDP at constant prices & 4.2 & 7.9 & 13.9 & 9.1 & 7.9 \\
\hline
\end{tabular}

Sources: Central Statistical Office; and Fund staff estimates. 
Table 4. Trinidad and Tobago: GDP by Final Expenditure at Current Market Prices

\begin{tabular}{|c|c|c|c|c|c|}
\hline & 2001 & 2002 & 2003 & 2004 & 2005 \\
\hline & \multicolumn{5}{|c|}{ (In millions of Trinidad and Tobago dollars) } \\
\hline Domestic expenditure & 53,517 & 57,548 & 67,568 & 71,671 & 73,974 \\
\hline Consumption & 35,492 & 42,821 & 46,722 & 55,342 & 56,483 \\
\hline Private sector & 29,351 & 35,572 & 37,197 & 44,388 & 44,241 \\
\hline Government & 6,141 & 7,249 & 9,526 & 10,954 & 12,242 \\
\hline Gross capital formation & 10,696 & 10,826 & 12,226 & 15,188 & 17,479 \\
\hline Private sector & 7,471 & 8,889 & 9,666 & 10,682 & 10,078 \\
\hline Government & 3,225 & 1,937 & 2,560 & 4,505 & 7,401 \\
\hline Net exports & 5,723 & 3,117 & 8,114 & 13,538 & 28,154 \\
\hline Exports of goods and nonfactor services & 30,244 & 28,299 & 34,173 & 45,550 & 67,213 \\
\hline Imports of goods and nonfactor services & 24,521 & 25,182 & 26,059 & 32,011 & 39,059 \\
\hline GDP at market prices & 59,239 & 60,665 & 75,681 & 85,209 & 102,128 \\
\hline Net factor payments & $-3,343$ & $-2,980$ & $-4,378$ & $-2,771$ & $-3,493$ \\
\hline Net transfers & 207 & 338 & 364 & 337 & 331 \\
\hline \multirow[t]{2}{*}{ GNP at market prices } & 56,103 & 58,023 & 71,667 & 82,775 & 98,966 \\
\hline & \multicolumn{5}{|c|}{ (In percent of GDP) } \\
\hline Domestic expenditure & 90.3 & 94.9 & 89.3 & 84.1 & 72.4 \\
\hline Consumption & 59.9 & 70.6 & 61.7 & 64.9 & 55.3 \\
\hline Private sector & 49.5 & 58.6 & 49.1 & 52.1 & 43.3 \\
\hline Government & 10.4 & 11.9 & 12.6 & 12.9 & 12.0 \\
\hline Gross capital formation & 18.1 & 17.8 & 16.2 & 17.8 & 17.1 \\
\hline Private sector & 12.6 & 14.7 & 12.8 & 12.5 & 9.9 \\
\hline Government & 5.4 & 3.2 & 3.4 & 5.3 & 7.2 \\
\hline Net exports & 9.7 & 5.1 & 10.7 & 15.9 & 27.6 \\
\hline Exports of goods and nonfactor services & 51.1 & 46.6 & 45.2 & 53.5 & 65.8 \\
\hline Imports of goods and nonfactor services & 41.4 & 41.5 & 34.4 & 37.6 & 38.2 \\
\hline GDP at market prices & 100.0 & 100.0 & 100.0 & 100.0 & 100.0 \\
\hline Net factor payments & -5.6 & -4.9 & -5.8 & -3.3 & -3.4 \\
\hline Net transfers & 0.3 & 0.6 & 0.5 & 0.4 & 0.3 \\
\hline \multirow[t]{2}{*}{ GNP at market prices } & 94.7 & 95.6 & 94.7 & 97.1 & 96.9 \\
\hline & \multicolumn{5}{|c|}{ (Contribution to growth) } \\
\hline Domestic expenditure & 18.1 & 6.8 & 16.5 & 5.4 & 2.7 \\
\hline Net exports & -2.7 & -4.4 & 8.2 & 7.2 & 17.2 \\
\hline GDP at constant prices & 15.3 & 2.4 & 24.8 & 12.6 & 19.9 \\
\hline
\end{tabular}

Sources: Central Statistical Office; and Fund staff estimates. 
Table 5. Trinidad and Tobago: Savings and Investment at Current Market Prices

\begin{tabular}{|c|c|c|c|c|c|}
\hline & 2001 & 2002 & 2003 & 2004 & 2005 \\
\hline & \multicolumn{5}{|c|}{ (In millions of Trinidad and Tobago dollars) } \\
\hline GDP at market prices & 59,239 & 60,665 & 75,681 & 85,209 & 102,128 \\
\hline Gross domestic savings & 16,419 & 13,942 & 20,340 & 28,726 & 45,632 \\
\hline Private sector & 14,522 & 13,181 & 16,732 & 22,901 & 35,982 \\
\hline Public sector & 1,897 & 762 & 3,608 & 5,825 & 9,650 \\
\hline Net factor payments & 3,343 & 2,980 & 4,378 & 2,771 & 3,493 \\
\hline Net transfers & -207 & -338 & -364 & -337 & -331 \\
\hline Gross national savings & 13,282 & 11,301 & 16,325 & 26,292 & 42,470 \\
\hline Private sector & 11,386 & 10,540 & 12,718 & 20,466 & 32,820 \\
\hline Public sector & 1,897 & 762 & 3,608 & 5,825 & 9,650 \\
\hline Government & 965 & 1,033 & 2,512 & 4,443 & 8,392 \\
\hline Public enterprises & 931 & -271 & 1,096 & 1,382 & 1,258 \\
\hline Gross domestic investment & 10,696 & 10,826 & 12,226 & 15,188 & 17,479 \\
\hline Private sector & 7,471 & 8,889 & 9,666 & 10,682 & 10,078 \\
\hline Public sector & 3,225 & 1,937 & 2,560 & 4,505 & 7,401 \\
\hline Government & 773 & 810 & 1,149 & 1,901 & 3,126 \\
\hline Public enterprises & 2,570 & 1,375 & 1,829 & 3,308 & 5,576 \\
\hline Investment-savings gap & $-2,586$ & -476 & $-4,099$ & $-11,104$ & $-24,992$ \\
\hline Private sector & $-3,914$ & $-1,651$ & $-3,052$ & $-9,784$ & $-22,742$ \\
\hline Public sector & 1,328 & 1,175 & $-1,048$ & $-1,320$ & $-2,250$ \\
\hline Government & -192 & -222 & $-1,363$ & $-2,542$ & $-5,267$ \\
\hline \multirow[t]{2}{*}{ Public enterprises } & 1,639 & 1,646 & 733 & 1,926 & 4,318 \\
\hline & \multicolumn{5}{|c|}{ (In percent of GDP) } \\
\hline Gross domestic savings & 27.7 & 23.0 & 26.9 & 33.7 & 44.7 \\
\hline Private sector & 24.5 & 21.7 & 22.1 & 26.9 & 35.2 \\
\hline Public sector & 3.2 & 1.3 & 4.8 & 6.8 & 9.4 \\
\hline Net factor payments & 5.6 & 4.9 & 5.8 & 3.3 & 3.4 \\
\hline Net transfers & -0.3 & -0.6 & -0.5 & -0.4 & -0.3 \\
\hline Gross national savings & 22.4 & 18.6 & 21.6 & 30.9 & 41.6 \\
\hline Private sector & 19.2 & 17.4 & 16.8 & 24.0 & 32.1 \\
\hline Public sector & 3.2 & 1.3 & 4.8 & 6.8 & 9.4 \\
\hline Government & 1.6 & 1.7 & 3.3 & 5.2 & 8.2 \\
\hline Public enterprises & 1.6 & -0.4 & 1.4 & 1.6 & 1.2 \\
\hline Gross domestic investment & 18.1 & 17.8 & 16.2 & 17.8 & 17.1 \\
\hline Private sector & 12.6 & 14.7 & 12.8 & 12.5 & 9.9 \\
\hline Public sector & 5.4 & 3.2 & 3.4 & 5.3 & 7.2 \\
\hline Government & 1.3 & 1.3 & 1.5 & 2.2 & 3.1 \\
\hline Public enterprises & 4.3 & 2.3 & 2.4 & 3.9 & 5.5 \\
\hline Investment-savings gap & -4.4 & -0.8 & -5.4 & -13.0 & -24.5 \\
\hline Private sector & -6.6 & -2.7 & -4.0 & -11.5 & -22.3 \\
\hline Public sector & 2.2 & 1.9 & -1.4 & -1.5 & -2.2 \\
\hline Government & -0.3 & -0.4 & -1.8 & -3.0 & -5.2 \\
\hline Public enterprises & 2.8 & 2.7 & 1.0 & 2.3 & 4.2 \\
\hline
\end{tabular}

Sources: Central Statistical Office; and Fund staff estimates. 
Table 6. Trinidad and Tobago: Retail Price Index

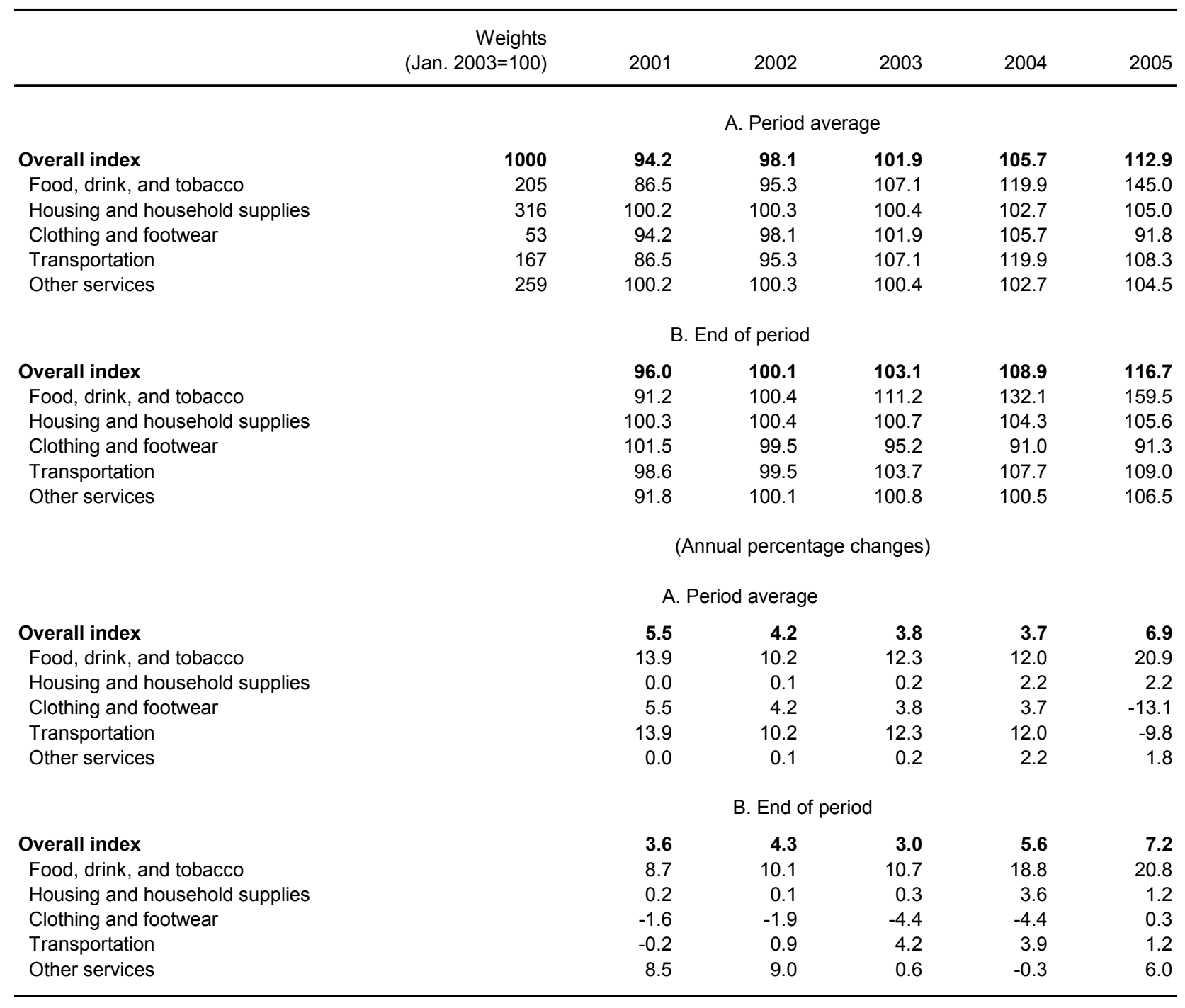

Sources: Central Statistical Office; and Fund staff calculations. 
Table 7. Trinidad and Tobago: Index of Producer Prices by Industry

\begin{tabular}{|c|c|c|c|c|c|c|}
\hline & Weights & 2001 & 2002 & 2003 & 2004 & 2005 \\
\hline & \multicolumn{6}{|c|}{ (Period averages: October 1978=100) } \\
\hline Producer prices & 1,000 & 363.9 & 366.1 & 371.3 & 382.3 & 391.0 \\
\hline Food processing & 191 & 420.6 & 425.2 & 443.0 & 453.8 & 459.3 \\
\hline Drink and tobacco & 121 & 479.5 & 505.8 & 515.8 & 544.1 & 573.8 \\
\hline Chemical and nonmetallic products & 148 & 415.7 & 417.1 & 422.2 & 424.9 & 435.2 \\
\hline Assembly type industry & 257 & 295.8 & 295.5 & 300.5 & 314.8 & 321.4 \\
\hline \multirow[t]{2}{*}{ Other } & 283 & 303.3 & 303.7 & 298.9 & 303.9 & 306.9 \\
\hline & \multicolumn{6}{|c|}{ (Annual percentage changes) } \\
\hline Producer prices & & 0.9 & 0.6 & 1.4 & 3.0 & 2.3 \\
\hline Food processing & & 0.3 & 1.1 & 4.2 & 2.4 & 1.2 \\
\hline Drink and tobacco & & -0.1 & 5.5 & 2.0 & 5.5 & 5.5 \\
\hline Chemical and nonmetallic products & & 0.0 & 0.3 & 1.2 & 0.6 & 2.4 \\
\hline Assembly type industry & & 0.6 & -0.1 & 1.7 & 4.8 & 2.1 \\
\hline Other & & 0.3 & 0.1 & -1.6 & 1.7 & 1.0 \\
\hline
\end{tabular}

Source: Central Statistical Office. 
Table 8. Trinidad and Tobago: Labor Force and Employment

\begin{tabular}{|c|c|c|c|c|c|}
\hline & 2001 & 2002 & 2003 & 2004 & 2005 \\
\hline & \multicolumn{5}{|c|}{ (In thousands) } \\
\hline Population & 1,267 & 1,276 & 1,282 & 1,291 & 1,295 \\
\hline \multicolumn{6}{|l|}{ Of which } \\
\hline 15 years and over & 949.9 & 961.8 & 968.3 & 973.6 & 979.0 \\
\hline Labor force & 576.5 & 586.2 & 596.5 & 613.5 & 623.7 \\
\hline Male & 356.7 & 356.9 & 360.4 & 365.4 & 364.9 \\
\hline Female & 219.8 & 229.3 & 236.1 & 248.1 & 258.8 \\
\hline Employed & 514.1 & 525.1 & 535.1 & 562.3 & 574.0 \\
\hline Male & 326.0 & 329.0 & 330.6 & 342.0 & 343.6 \\
\hline \multirow[t]{2}{*}{ Female } & 188.1 & 196.1 & 203.5 & 220.3 & 230.4 \\
\hline & \multicolumn{5}{|c|}{ (As a percentage of the labor force) } \\
\hline Unemployed & 10.8 & 10.4 & 10.3 & 8.3 & 8.0 \\
\hline Seeking work & 7.5 & 7.3 & 7.2 & 5.9 & 5.6 \\
\hline Other unemployed & 3.3 & 3.1 & 3.1 & 2.4 & 2.4 \\
\hline
\end{tabular}

Source: Central Statistical Office. 
Table 9. Trinidad and Tobago: Growth of Production, Earnings, Employment, Hours and Costs in Manufacturing 1/

\begin{tabular}{|c|c|c|c|c|c|}
\hline & 2001 & 2002 & 2003 & 2004 & 2005 \\
\hline & \multicolumn{5}{|c|}{ (Annual percentage changes) } \\
\hline \multicolumn{6}{|l|}{ All industry } \\
\hline Production & 11.5 & 15.0 & 9.5 & 6.1 & 9.8 \\
\hline Weekly earnings & 9.3 & 11.6 & 13.2 & 14.5 & 1.4 \\
\hline Productivity per man/hour & 12.0 & 11.9 & 12.1 & 3.7 & 7.6 \\
\hline Employment & 3.0 & -1.2 & 0.0 & 1.9 & 3.4 \\
\hline Hours worked & -0.6 & 2.7 & -2.2 & 2.2 & 2.0 \\
\hline Earnings per man/hour & 10.0 & 8.7 & 15.9 & 12.3 & -0.1 \\
\hline Unit labor cost & -2.6 & -2.7 & 3.3 & 8.1 & $\ldots$ \\
\hline \multicolumn{6}{|c|}{ Manufacturing (Excluding oil and sugar) } \\
\hline Production & 20.7 & 11.8 & 10.9 & 12.5 & 11.1 \\
\hline Weekly earnings & 9.3 & 7.1 & 9.8 & 11.2 & 3.9 \\
\hline Productivity per man/hour & 25.3 & 9.0 & 16.4 & 9.8 & 7.3 \\
\hline Employment & -3.1 & 5.0 & -3.5 & 3.4 & -0.2 \\
\hline Hours worked & -3.8 & 2.4 & -4.5 & 2.3 & 3.6 \\
\hline Earnings per man/hour & 13.7 & 4.5 & 15.0 & 9.1 & 0.3 \\
\hline Unit labor cost & -9.7 & -4.0 & -0.7 & -0.5 & \\
\hline \multicolumn{6}{|l|}{ Oil refining } \\
\hline Production & 4.5 & 5.1 & 12.8 & 2.5 & 28.6 \\
\hline Weekly earnings & 11.4 & 12.7 & 13.4 & 21.5 & -0.3 \\
\hline Productivity per man/hour & -6.2 & 4.6 & 6.4 & 4.7 & 26.9 \\
\hline Employment & 10.2 & -10.1 & -1.7 & 3.1 & 3.7 \\
\hline Hours worked & 10.8 & -0.4 & 6.1 & -1.9 & 1.4 \\
\hline Earnings per man/hour & 0.9 & 13.4 & 6.8 & 24.1 & -1.7 \\
\hline Unit labor cost & 6.6 & 7.2 & 0.2 & 19.5 & $\ldots$ \\
\hline
\end{tabular}

Sources: Central Statistical Office; and Fund staff estimates.

1/ All employees. 
Table 10. Trinidad and Tobago: Central Government Operations 1/

\begin{tabular}{|c|c|c|c|c|c|}
\hline & $2000 / 01$ & $2001 / 02$ & $2002 / 03$ & $2003 / 04$ & $2004 / 05$ \\
\hline & \multicolumn{5}{|c|}{ (In millions of Trinidad and Tobago dollars) } \\
\hline Total revenue and grants & 14,200 & 13,860 & 17,350 & 20,629 & 29,649 \\
\hline Energy sector & 4,951 & 3,273 & 6,720 & 8,484 & 15,614 \\
\hline Nonenergy sector & 9,211 & 10,553 & 10,626 & 12,141 & 14,026 \\
\hline Capital revenue and grants & 38 & 35 & 4 & 4 & 9 \\
\hline Total expenditure & 13,860 & 13,961 & 16,048 & 19,068 & 24,141 \\
\hline Current & 13,078 & 13,214 & 15,050 & 17,466 & 21,342 \\
\hline Wages and salaries & 4,284 & 4,225 & 4,548 & 4,849 & 5,309 \\
\hline Other goods and services & 1,768 & 1,742 & 2,038 & 2,375 & 3,172 \\
\hline Interest payments & 2,330 & 2,260 & 2,592 & 2,364 & 2,542 \\
\hline Transfer and subsidies & 4,697 & 4,988 & 5,872 & 7,878 & 10,320 \\
\hline Capital expenditure and net lending & 782 & 747 & 998 & 1,601 & 2,799 \\
\hline Current balance & 1,083 & 611 & 2,296 & 3,158 & 8,298 \\
\hline Overall balance & 339 & -101 & 1,302 & 1,561 & 5,508 \\
\hline Total financing & -339 & 101 & $-1,302$ & $-1,561$ & $-5,508$ \\
\hline Foreign financing & -2 & -194 & 12 & -357 & $-1,121$ \\
\hline Domestic financing & -338 & 295 & $-1,313$ & $-1,204$ & $-4,235$ \\
\hline \multirow[t]{2}{*}{ Of which: Revenue Stabilization Fund } & -546 & -600 & -497 & $-1,263$ & $-2,593$ \\
\hline & \multicolumn{5}{|c|}{ (In percent of GDP) } \\
\hline Total revenue & 24.8 & 23.0 & 24.1 & 24.9 & 30.3 \\
\hline Energy sector & 8.6 & 5.4 & 9.3 & 10.2 & 15.9 \\
\hline Nonenergy sector & 16.1 & 17.5 & 14.8 & 14.7 & 14.3 \\
\hline Capital revenue and grants & 0.1 & 0.1 & 0.0 & 0.0 & 0.0 \\
\hline Total expenditure & 24.2 & 23.1 & 22.3 & 23.0 & 24.7 \\
\hline Current & 22.8 & 21.9 & 20.9 & 21.1 & 21.8 \\
\hline Wages and salaries & 7.5 & 7.0 & 6.3 & 5.9 & 5.4 \\
\hline Other goods and services & 3.1 & 2.9 & 2.8 & 2.9 & 3.2 \\
\hline Interest payments & 4.1 & 3.7 & 3.6 & 2.9 & 2.6 \\
\hline Transfer and subsidies & 8.2 & 8.3 & 8.2 & 9.5 & 10.5 \\
\hline Capital expenditure and net lending & 1.4 & 1.2 & 1.4 & 1.9 & 2.9 \\
\hline Current balance & 1.9 & 1.0 & 3.2 & 3.8 & 8.5 \\
\hline Overall balance & 0.6 & -0.2 & 1.8 & 1.9 & 5.6 \\
\hline Total financing & -0.6 & 0.2 & -1.8 & -1.9 & -5.6 \\
\hline External financing & 0.0 & -0.3 & 0.0 & -0.4 & -1.1 \\
\hline Domestic financing & -0.6 & 0.5 & -1.8 & -1.5 & -4.3 \\
\hline Of which: Revenue Stabilization Fund & -1.0 & -1.0 & -0.7 & -1.5 & -2.6 \\
\hline
\end{tabular}

Sources: Ministry of Finance; and Fund staff estimates.

1/ Fiscal year basis. 
Table 11. Trinidad and Tobago: Central Government Revenue and Grants (In millions of Trinidad and Tobago dollars)

\begin{tabular}{|c|c|c|c|c|c|}
\hline & $2000 / 01$ & $2001 / 02$ & $2002 / 03$ & $2003 / 04$ & $2004 / 05$ \\
\hline Total revenue and grants & 14,200 & 13,860 & 17,350 & 20,629 & 29,649 \\
\hline Energy sector & 4,951 & 3,273 & 6,720 & 8,484 & 15,614 \\
\hline Corporation tax & 3,769 & 2,182 & 5,028 & 6,515 & 12,636 \\
\hline Business levy & 90 & 59 & 74 & 109 & 146 \\
\hline Royalties & 752 & 613 & 1,008 & 1,095 & 1,231 \\
\hline Unemployment levy & 191 & 124 & 294 & 410 & 905 \\
\hline Signature bonuses & 0 & 128 & 31 & 0 & 0 \\
\hline Impost & 23 & 30 & 34 & 37 & 43 \\
\hline Withholding tax (oil) & 126 & 88 & 158 & 178 & 467 \\
\hline Green Fund levy & 0 & 50 & 94 & 139 & 186 \\
\hline Nonenergy sector & 9,211 & 10,553 & 10,626 & 12,141 & 14,026 \\
\hline Taxes & 8,020 & 9,028 & 9,538 & 10,914 & 12,252 \\
\hline Income tax & 3,547 & 4,101 & 4,671 & 4,876 & 5,857 \\
\hline Goods and services & 3,425 & 3,817 & 3,546 & 4,438 & 4,537 \\
\hline VAT & 2,194 & 2,475 & 2,121 & 3,021 & 2,963 \\
\hline Excise duties on petrol & 494 & 525 & 563 & 587 & 635 \\
\hline Other $1 /$ & 737 & 817 & 862 & 830 & 940 \\
\hline International trade & 857 & 879 & 1,070 & 1,288 & 1,527 \\
\hline Import duties & 811 & 854 & 1,019 & 1,233 & 1,463 \\
\hline Other & 46 & 25 & 51 & 55 & 64 \\
\hline Property & 59 & 94 & 78 & 85 & 63 \\
\hline Other 2/ & 132 & 137 & 175 & 226 & 268 \\
\hline Nontaxes & 1,192 & 1,525 & 1,088 & 1,227 & 1,773 \\
\hline Fees, rentals, etc. & 695 & 639 & 426 & 431 & 549 \\
\hline Public enterprise profit & 157 & 550 & 391 & 614 & 936 \\
\hline Central bank profit & 81 & 135 & 144 & 96 & 159 \\
\hline Interest receipts & 259 & 201 & 127 & 86 & 130 \\
\hline Capital revenue and grants & 38 & 35 & 4 & 4 & 9 \\
\hline \multicolumn{6}{|l|}{ Memorandum item: } \\
\hline Total tax revenue & 12,970 & 12,300 & 16,258 & 19,398 & 27,867 \\
\hline
\end{tabular}

Source: Ministry of Finance.

1/ Includes all other taxes on goods and services except for the port and airport departure taxes. 2/ Stamp duties and social security contributions. 
Table 12. Trinidad and Tobago: Ratios of Central Government Revenue and Grants

\begin{tabular}{|c|c|c|c|c|c|}
\hline & $2000 / 01$ & $2001 / 02$ & $2002 / 03$ & $2003 / 04$ & $2004 / 05$ \\
\hline & \multicolumn{5}{|c|}{ (In percent of GDP) } \\
\hline Total revenue and grants & 24.8 & 23.0 & 24.1 & 24.9 & 30.3 \\
\hline Energy sector & 8.6 & 5.4 & 9.3 & 10.2 & 15.9 \\
\hline Of which: corporation tax & 6.6 & 3.6 & 7.0 & 7.9 & 12.9 \\
\hline Of which: royalties & 1.3 & 1.0 & 1.4 & 1.3 & 1.3 \\
\hline Nonenergy & 16.1 & 17.5 & 14.8 & 14.7 & 14.3 \\
\hline Taxes & 14.0 & 15.0 & 13.3 & 13.2 & 12.5 \\
\hline Income & 6.2 & 6.8 & 6.5 & 5.9 & 6.0 \\
\hline Goods and services & 6.0 & 6.3 & 4.9 & 5.4 & 4.6 \\
\hline VAT & 3.8 & 4.1 & 2.9 & 3.6 & 3.0 \\
\hline Excise duties on petrol & 0.9 & 0.9 & 0.8 & 0.7 & 0.6 \\
\hline Other & 1.3 & 1.4 & 1.2 & 1.0 & 1.0 \\
\hline International trade & 1.5 & 1.5 & 1.5 & 1.6 & 1.6 \\
\hline Import duties & 1.4 & 1.4 & 1.4 & 1.5 & 1.5 \\
\hline Other & 0.1 & 0.0 & 0.1 & 0.1 & 0.1 \\
\hline Property & 0.1 & 0.2 & 0.1 & 0.1 & 0.1 \\
\hline Other & 0.2 & 0.2 & 0.2 & 0.3 & 0.3 \\
\hline Nontaxes & 2.1 & 2.5 & 1.5 & 1.5 & 1.8 \\
\hline \multirow[t]{2}{*}{ Capital revenue and grants } & 0.1 & 0.1 & 0.0 & 0.0 & 0.0 \\
\hline & \multicolumn{5}{|c|}{ (In percent of total revenue and grants) } \\
\hline Total revenue and grants & 100.0 & 100.0 & 100.0 & 100.0 & 100.0 \\
\hline Energy sector & 34.9 & 23.6 & 38.7 & 41.1 & 52.7 \\
\hline Of which : corporation tax & 26.5 & 15.7 & 29.0 & 31.6 & 42.6 \\
\hline Of which: royalties & 5.3 & 4.4 & 5.8 & 5.3 & 4.2 \\
\hline Nonenergy & 64.9 & 76.1 & 61.2 & 58.9 & 47.3 \\
\hline Taxes & 56.5 & 65.1 & 55.0 & 52.9 & 41.3 \\
\hline Income & 25.0 & 29.6 & 26.9 & 23.6 & 19.8 \\
\hline Goods and services & 24.1 & 27.5 & 20.4 & 21.5 & 15.3 \\
\hline VAT & 15.4 & 17.9 & 12.2 & 14.6 & 10.0 \\
\hline Excise duties on petrol & 3.5 & 3.8 & 3.2 & 2.8 & 2.1 \\
\hline Other $1 /$ & 5.2 & 5.9 & 5.0 & 4.0 & 3.2 \\
\hline International trade & 6.0 & 6.3 & 6.2 & 6.2 & 5.1 \\
\hline Import duties & 5.7 & 6.2 & 5.9 & 6.0 & 4.9 \\
\hline Other & 0.3 & 0.2 & 0.3 & 0.3 & 0.2 \\
\hline Property & 0.4 & 0.7 & 0.4 & 0.4 & 0.2 \\
\hline Other 2/ & 0.9 & 1.0 & 1.0 & 1.1 & 0.9 \\
\hline Nontaxes & 8.4 & 11.0 & 6.3 & 5.9 & 6.0 \\
\hline Capital revenue and grants & 0.3 & 0.3 & 0.0 & 0.0 & 0.0 \\
\hline \multicolumn{6}{|l|}{ Memorandum item: } \\
\hline Tax revenue & 91.6 & 89.0 & 93.7 & 94.1 & 94.0 \\
\hline
\end{tabular}

Source: Ministry of Finance.

$1 /$ Includes all other taxes on goods and services except for the port and airport departure taxes. 2/ Stamp duties and social security contributions. 
Table 13. Trinidad and Tobago: Central Government Expenditure (In millions of Trinidad and Tobago dollars)

\begin{tabular}{|c|c|c|c|c|c|}
\hline & $2000 / 01$ & $2001 / 02$ & $2002 / 03$ & $2003 / 04$ & $2004 / 05$ \\
\hline Total expenditure & 13,860 & 13,961 & 16,048 & 19,068 & 24,141 \\
\hline Current & 13,078 & 13,214 & 15,050 & 17,466 & 21,342 \\
\hline Wages and salaries $1 /$ & 4,284 & 4,225 & 4,548 & 4,849 & 5,309 \\
\hline Other goods and services & 1,768 & 1,742 & 2,038 & 2,375 & 3,172 \\
\hline Interest payments & 2,330 & 2,260 & 2,592 & 2,364 & 2,542 \\
\hline Domestic 2/ & 1,440 & 1,459 & 1,834 & 1,638 & 1,876 \\
\hline External & 890 & 800 & 758 & 726 & 666 \\
\hline Transfers and subsidies & 4,697 & 4,988 & 5,872 & 7,878 & 10,320 \\
\hline Public sector $3 /$ & 1,538 & 1,616 & 1,914 & 3,035 & 3,566 \\
\hline Local government and Tobago & 966 & 1,043 & 1,150 & 1,471 & 1,520 \\
\hline Utilities, state enterprises & 571 & 573 & 764 & 1,564 & 2,046 \\
\hline Households & 1,666 & 1,906 & 2,023 & 2,131 & 2,582 \\
\hline Other 4/ & 1,493 & 1,465 & 1,935 & 2,722 & 4,172 \\
\hline Capital expenditure and net lending & 782 & 747 & 998 & 1,601 & 2,799 \\
\hline Capital expenditure & 1,027 & 983 & 1,227 & 1,841 & 3,076 \\
\hline Capital transfers & 0 & 1 & 2 & 3 & 4 \\
\hline Net lending & -245 & -236 & -229 & -240 & -278 \\
\hline \multicolumn{6}{|l|}{ Memorandum item: } \\
\hline Noninterest spending & 11,531 & 11,702 & 13,456 & 16,703 & 21,599 \\
\hline
\end{tabular}

Source: Ministry of Finance.

$1 /$ Includes contributions to the National Insurance Board and severance payments to central government employees.

2/ Includes interest on domestic loans denominated in U.S. dollars and debt service on DFCs.

3/ Statutory bodies, state enterprises (including public utilities), and local governments.

4/ Comprises subsidies, nonprofit institutions, statutory authorities, transfers abroad, and other transfers (excluding Revenue Stabilization Fund). 
Table 14. Trinidad and Tobago: Central Government Expenditure Ratios

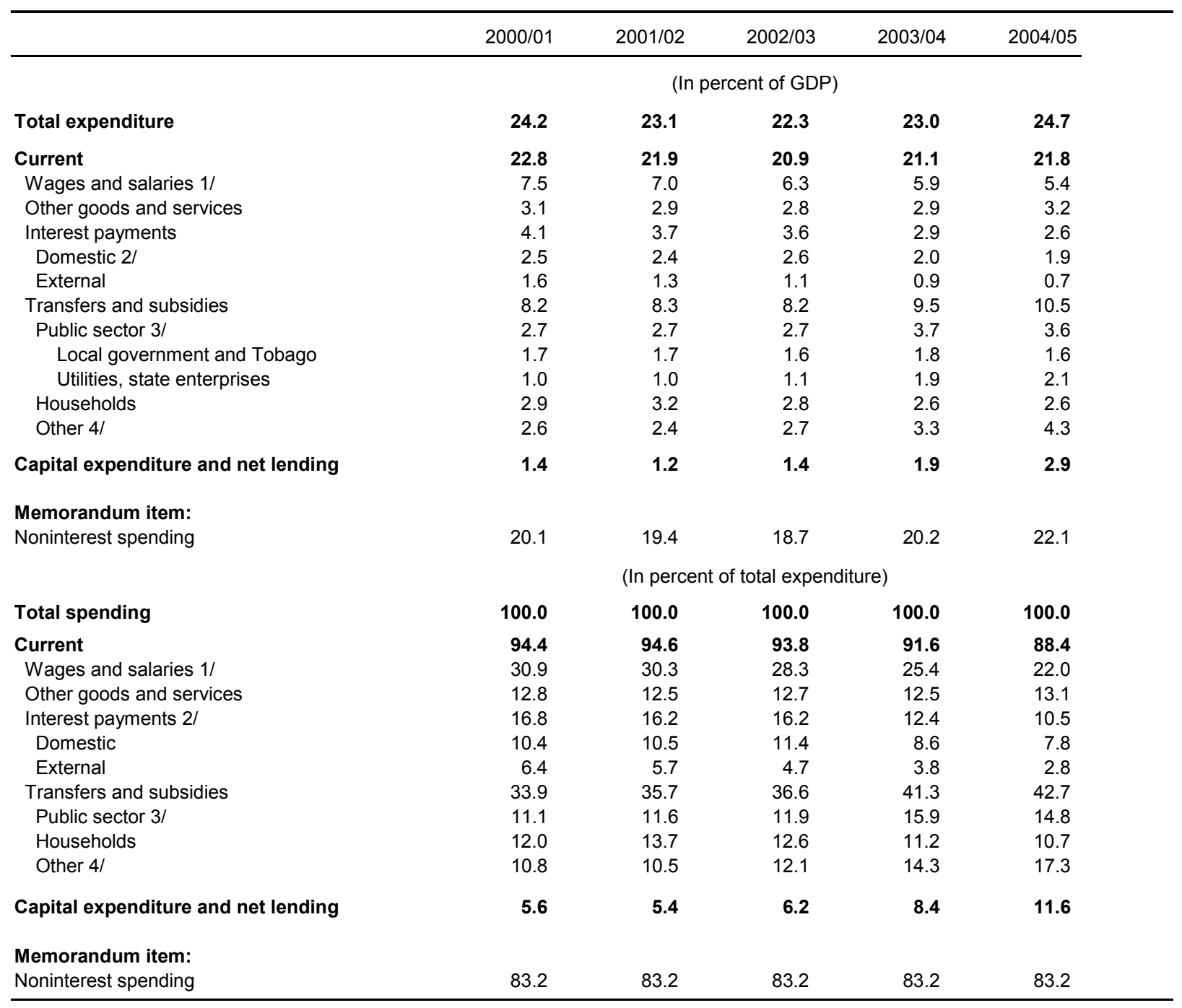

Source: Ministry of Finance.

1/ Includes contributions to the National Insurance Board and severance payments to central government employees.

2/ Includes interest on domestic loans denominated in U.S. dollars and debt service on DFCs.

$3 /$ Statutory bodies, state enterprises (including public utilities), and local governments.

4/ Comprises subsidies, nonprofit institutions, statutory authorities, transfers abroad, and other transfers (excluding Revenue Stabilizati 
Table 15a. Trinidad and Tobago: Public Enterprises

\begin{tabular}{|c|c|c|c|c|c|}
\hline & $2000 / 01$ & $2001 / 02$ & $2002 / 03$ & $2003 / 04$ & $2004 / 05$ \\
\hline & \multicolumn{5}{|c|}{ (In millions of Trinidad and Tobago dollars) } \\
\hline Total revenues & 21,654 & 17,251 & 23,288 & 25,462 & 35,199 \\
\hline Operating revenues & 21,330 & 17,041 & 22,994 & 24,442 & 34,327 \\
\hline Capital revenue and grants & 324 & 210 & 294 & 1,020 & 872 \\
\hline Energy related SOEs & 18,209 & 13,714 & 19,513 & 22,249 & 31,523 \\
\hline Nonenergy SOEs & 3,445 & 3,537 & 3,775 & 3,212 & 3,676 \\
\hline Current expenditures & 20,325 & 17,670 & 22,033 & 22,784 & 33,238 \\
\hline Wages and salaries & 2,218 & 2,323 & 3,000 & 2,501 & 2,887 \\
\hline Of which: severance payments & 63 & 74 & 747 & 9 & 13 \\
\hline Other goods and services & 14,749 & 13,546 & 16,172 & 16,939 & 24,580 \\
\hline Interest payments & 1,150 & 517 & 628 & 604 & 507 \\
\hline Domestic & 328 & 276 & 439 & 387 & 345 \\
\hline External & 822 & 241 & 189 & 216 & 161 \\
\hline Other & 383 & 272 & 271 & 404 & 262 \\
\hline Taxes and dividends & 1,826 & 1,012 & 1,963 & 2,337 & 5,002 \\
\hline Operating balance & 1,004 & -629 & 960 & 1,657 & 1,089 \\
\hline Energy related SOEs & 1,165 & -390 & 2,052 & 2,182 & 1,062 \\
\hline Nonenergy SOEs & -161 & -239 & $-1,091$ & -525 & 27 \\
\hline Government transfers & 553 & 337 & 589 & 1,003 & 1,257 \\
\hline Current & 296 & 233 & 388 & 636 & 705 \\
\hline Capital & 257 & 103 & 201 & 367 & 552 \\
\hline Capital expenditures and net lending & 2,511 & 1,288 & 1,475 & 2,872 & 4,418 \\
\hline Energy related SOEs & 1,709 & 429 & 858 & 1,923 & 2,472 \\
\hline Nonenergy SOEs & 575 & 637 & 468 & 764 & 1,635 \\
\hline Net lending & 228 & 221 & 149 & 184 & 312 \\
\hline Overall balance & -630 & $-1,370$ & 368 & 808 & $-1,200$ \\
\hline Energy related SOEs & -332 & -827 & 1,339 & 1,138 & -828 \\
\hline Nonenergy public enterprises & -298 & -543 & -971 & -330 & -372 \\
\hline Statistical discrepancy & -167 & 150 & -307 & -424 & -544 \\
\hline Identified financing & 463 & 1,520 & -675 & $-1,232$ & 656 \\
\hline Net external financing & 441 & 845 & -257 & $-2,503$ & 390 \\
\hline Disbursements & 6,356 & 3,351 & 2,063 & 3,997 & 6,590 \\
\hline Amortization & $-5,915$ & $-2,506$ & $-2,320$ & $-6,500$ & $-6,201$ \\
\hline Net domestic financing & 22 & 675 & -418 & 1,271 & 266 \\
\hline Disbursements & 5,686 & 3,798 & 3,379 & 4,209 & 5,009 \\
\hline \multirow[t]{2}{*}{ Amortization } & $-5,664$ & $-3,123$ & $-3,797$ & $-2,938$ & $-4,743$ \\
\hline & \multicolumn{4}{|c|}{ (In percent of GDP) } & \\
\hline Total revenues & 37.8 & 28.6 & 32.4 & 30.7 & 36.0 \\
\hline Energy related SOEs & 31.8 & 22.7 & 27.1 & 26.9 & 32.2 \\
\hline Nonenergy SOEs & 6.0 & 5.9 & 5.2 & 3.9 & 3.8 \\
\hline Current expenditures & 35.5 & 29.3 & 30.6 & 27.5 & 34.0 \\
\hline Wages and salaries & 3.9 & 3.9 & 4.2 & 3.0 & 2.9 \\
\hline Other goods and services & 25.8 & 22.5 & 22.5 & 20.5 & 25.1 \\
\hline Interest payments & 2.0 & 0.9 & 0.9 & 0.7 & 0.5 \\
\hline Other & 0.6 & 0.5 & 0.6 & 0.5 & 0.4 \\
\hline Taxes and dividends & 1.4 & 0.4 & 0.3 & 0.3 & 0.2 \\
\hline Operating balance & 1.8 & -1.0 & 1.3 & 2.0 & 1.1 \\
\hline Energy related SOEs & 2.0 & -0.6 & 2.9 & 2.6 & 1.1 \\
\hline Nonenergy SOEs & -0.3 & -0.4 & -1.5 & -0.6 & 0.0 \\
\hline Government transfers & 1.0 & 0.6 & 0.8 & 1.2 & 1.3 \\
\hline Capital expenditures & 4.4 & 2.1 & 2.1 & 3.5 & 4.5 \\
\hline Energy related SOEs & 3.0 & 0.7 & 1.2 & 2.3 & 2.5 \\
\hline Nonenergy SOEs & 1.0 & 1.1 & 0.7 & 0.9 & 1.7 \\
\hline Net lending & 0.4 & 0.4 & 0.2 & 0.2 & 0.3 \\
\hline Overall balance & -1.1 & -2.3 & 0.5 & 1.0 & -1.2 \\
\hline Energy related SOEs & -0.6 & -1.4 & 1.9 & 1.4 & -0.8 \\
\hline Nonenergy public enterprises & -0.5 & -0.9 & -1.3 & -0.4 & -0.4 \\
\hline \multicolumn{6}{|l|}{ Memorandum item: } \\
\hline Nominal GDP (in TT\$ million, Fiscal year) & 57,272 & 60,308 & 71,927 & 82,827 & 97,898 \\
\hline
\end{tabular}

Sources: Ministry of Finance; and Fund staff estimates and projection .

1/ Comprises the major enterprises namely CARONI, MTS, NFM, NFM, NHSL, NPMC, NQCL, NGC, PETROTRIN, PLIPDECO, SWMCOL, TIDCO, TANTEAK,TRINGEN, TTST, and UDECOTT. Data for National Housing Authority was not available. 
Table 15b. Trinidad and Tobago: Statutory Bodies 1/

\begin{tabular}{|c|c|c|c|c|c|}
\hline & $2000 / 01$ & $2001 / 02$ & $2002 / 03$ & $2003 / 04$ & $2004 / 05$ \\
\hline & \multicolumn{5}{|c|}{ (In millions of Trinidad and Tobago dollars) } \\
\hline Total revenues & 2,173 & 2,005 & 2,339 & 2,393 & 2,506 \\
\hline Operating revenues & 2,085 & 2,005 & 2,333 & 2,392 & 2,506 \\
\hline Capital revenue and grants & 88 & 0 & 6 & 1 & 0 \\
\hline $\begin{array}{l}\text { Current expenditures } \\
\text { Wages and salaries }\end{array}$ & $\begin{array}{r}2,336 \\
677\end{array}$ & $\begin{array}{r}2,510 \\
716\end{array}$ & $\begin{array}{r}2,995 \\
806\end{array}$ & $\begin{array}{l}3,427 \\
1,024\end{array}$ & $\begin{array}{l}3,659 \\
1,157\end{array}$ \\
\hline Other goods and services & 1,403 & 1,409 & 2,045 & 2,197 & 2,276 \\
\hline Interest payments & 307 & 366 & 412 & 437 & 614 \\
\hline Other & 21 & 76 & 22 & 18 & 20 \\
\hline Taxes and dividends & -73 & -58 & -289 & -249 & -408 \\
\hline \multirow[t]{2}{*}{ Operating balance } & -252 & -505 & -662 & $-1,035$ & $-1,153$ \\
\hline & 0 & 0 & 0 & 0 & \\
\hline Government transfers & 286 & 324 & 441 & 448 & 932 \\
\hline Current & 207 & 219 & 275 & 244 & 382 \\
\hline Capital & 78 & 105 & 167 & 204 & 550 \\
\hline Capital expenditures and net lending & 725 & 275 & 191 & 203 & 633 \\
\hline Capital expenditures & 696 & 275 & 149 & 203 & 452 \\
\hline Net lending & 28 & 0 & 42 & 0 & 181 \\
\hline Overall balance & -602 & -456 & -406 & -790 & -854 \\
\hline Statistical discrepancy & -379 & -75 & -83 & -161 & -244 \\
\hline Identified financing & 224 & 380 & 323 & 629 & 609 \\
\hline Net external financing & 18 & -49 & -55 & -64 & -164 \\
\hline Disbursements & 57 & 0 & 0 & 0 & 0 \\
\hline Amortization & -39 & -49 & -55 & -64 & -164 \\
\hline Net domestic financing & 205 & 430 & 378 & 693 & 773 \\
\hline Disbursements & 335 & 875 & 658 & 1,085 & 1,653 \\
\hline \multirow[t]{2}{*}{ Amortization } & -130 & -446 & -280 & -392 & -880 \\
\hline & \multicolumn{5}{|c|}{ (In percent of GDP) } \\
\hline Total revenues & 3.8 & 3.3 & 3.3 & 2.9 & 2.6 \\
\hline Current expenditures & 4.1 & 4.2 & 4.2 & 4.1 & 3.7 \\
\hline Wages and salaries & 1.2 & 1.2 & 1.1 & 1.2 & 1.2 \\
\hline Other goods and services & 2.5 & 2.3 & 2.8 & 2.7 & 2.3 \\
\hline Interest payments & 0.5 & 0.6 & 0.6 & 0.5 & 0.6 \\
\hline Other & 0.0 & 0.1 & 0.0 & 0.0 & 0.0 \\
\hline Taxes and dividends & -0.1 & -0.1 & -0.4 & -0.3 & -0.4 \\
\hline Operating balance & -0.4 & -0.8 & -0.9 & -1.3 & -1.2 \\
\hline Government transfers & 0.5 & 0.5 & 0.6 & 0.5 & 1.0 \\
\hline Capital expenditures & 1.3 & 0.5 & 0.3 & 0.2 & 0.6 \\
\hline Capital & 1.2 & 0.5 & 0.2 & 0.2 & 0.5 \\
\hline Net lending & 0.0 & 0.0 & 0.1 & 0.0 & 0.2 \\
\hline Overall balance & -1.1 & -0.8 & -0.6 & -1.0 & -0.9 \\
\hline \multicolumn{6}{|l|}{ Memorandum item: } \\
\hline Nominal GDP (in TT\$ million, FY) & 57,272 & 60,308 & 71,927 & 82,827 & 97,898 \\
\hline
\end{tabular}

Sources: Ministry of Finance; and Fund staff estimates and projections

1/ Coverage includes Public Transport Company, Electricity Company, Water Authority, Airport Authority and Port Authority. 
Table 16. Trinidad and Tobago: Consolidated Nonfinancial Public Sector (In percent of GDP)

\begin{tabular}{|c|c|c|c|c|c|}
\hline & $2000 / 01$ & $2001 / 02$ & $2002 / 03$ & $2003 / 04$ & $2004 / 05$ \\
\hline & \multicolumn{4}{|c|}{ I. Nonfinancial Public Sector } & \\
\hline Current balance & 3.2 & -0.9 & 3.6 & 4.6 & 8.4 \\
\hline Capital revenue and transfers & 2.3 & 1.5 & 1.9 & 3.0 & 3.1 \\
\hline Capital expenditure and net lending & 7.0 & 3.8 & 3.7 & 5.6 & 8.0 \\
\hline Overall balance & -1.6 & -3.2 & 1.8 & 1.9 & 3.5 \\
\hline \multirow[t]{2}{*}{ Public sector debt 1/ 2/ } & 53.4 & 54.8 & 49.9 & 42.8 & 36.1 \\
\hline & \multicolumn{4}{|c|}{ II. Central Government } & \\
\hline Current balance & 1.9 & 1.0 & 3.2 & 3.8 & 8.5 \\
\hline Current revenue & 24.7 & 22.9 & 24.1 & 24.9 & 30.3 \\
\hline Current expenditure & 22.8 & 21.9 & 20.9 & 21.1 & 21.8 \\
\hline Capital revenue and grants & 0.1 & 0.1 & 0.0 & 0.0 & 0.0 \\
\hline Capital expenditure and net lending & 1.4 & 1.2 & 1.4 & 1.9 & 2.9 \\
\hline Overall balance & 0.6 & -0.2 & 1.8 & 1.9 & 5.6 \\
\hline \multirow[t]{2}{*}{ Central government debt 3 / } & 37.1 & 35.1 & 30.3 & 24.8 & 20.2 \\
\hline & \multicolumn{4}{|c|}{ III. Statutory Bodies 4/ } & \\
\hline Current balance & -0.4 & -0.8 & -0.9 & -1.3 & -1.2 \\
\hline Current revenue & 3.6 & 3.3 & 3.2 & 2.9 & 2.6 \\
\hline Current expenditure & 4.1 & 4.2 & 4.2 & 4.1 & 3.7 \\
\hline Capital revenue and transfers & 0.7 & 0.5 & 0.6 & 0.5 & 1.0 \\
\hline Capital expenditure and net lending & 1.3 & 0.5 & 0.3 & 0.2 & 0.6 \\
\hline Overall balance & -1.1 & -0.8 & -0.6 & -1.0 & -0.9 \\
\hline \multirow[t]{2}{*}{ Debt 3/ } & 8.8 & 8.5 & 7.7 & 8.0 & 7.8 \\
\hline & \multicolumn{4}{|c|}{ IV. Public Enterprises 5/ } & \\
\hline Current balance & 1.8 & -1.0 & 1.3 & 2.0 & 1.1 \\
\hline Current revenue & 37.2 & 28.3 & 32.0 & 29.5 & 35.1 \\
\hline Current expenditure & 35.5 & 29.3 & 30.6 & 27.5 & 34.0 \\
\hline Capital revenue and transfers & 1.5 & 0.9 & 1.2 & 2.4 & 2.2 \\
\hline Capital expenditure and net lending & 4.4 & 2.1 & 2.1 & 3.5 & 4.5 \\
\hline Overall balance & -1.1 & -2.3 & 0.5 & 1.0 & -1.2 \\
\hline Debt 3/ & 7.5 & 11.2 & 11.9 & 10.0 & 8.1 \\
\hline \multicolumn{6}{|l|}{ Memorandum item: } \\
\hline Nominal GDP (in TT\$ million, FY) & 57,272 & 60,308 & 71,927 & 82,827 & 97,898 \\
\hline
\end{tabular}

Sources: Ministry of Finance; and Fund staff estimates and projections.

1/ Refers to gross debt. The baseline projections assume a significant increase in government deposits at the CBTT, in the context of the Interim Revenue Stabilization Fund, and accordingly lower net debt.

2/ Includes BOLT and leases.

3/ Refers to gross debt, and it includes government guaranteed debt and letters of comfort.

4/ Includes Public Transport Company, Electricity Company, Water Authority, Airport Authority and Port Authority.

5/ Includes CARONI, MTS, NFM, NFM, NHSL, NPMC, NQCL, NGC, PETROTRIN, PLIPDECO, SWMCOL, TIDCO,

TANTEAK, TRINGEN, TTST, and UDECOTT. Data for National Housing Authority was not available. 
Table 17. Trinidad and Tobago: Summary Accounts of the Consolidated Financial System 1/

\begin{tabular}{|c|c|c|c|c|c|}
\hline & 2001 & 2002 & 2003 & 2004 & 2005 \\
\hline Net foreign assets & 12,875 & 13,607 & 15,435 & 25,087 & 35,918 \\
\hline Net international reserves & 11,832 & 12,317 & 13,584 & 21,459 & 32,174 \\
\hline Other foreign assets (net) & 1,043 & 1,290 & 1,851 & 3,628 & 3,744 \\
\hline Net domestic assets & 11,888 & 10,566 & 10,566 & 5,858 & 2,977 \\
\hline Credit to public sector & $-2,137$ & $-1,527$ & $-3,039$ & $-8,252$ & $-14,709$ \\
\hline Central government & $-2,709$ & $-3,088$ & $-4,263$ & $-9,509$ & $-16,144$ \\
\hline Rest of the public sector & 572 & 1,561 & 1,224 & 1,257 & 1,435 \\
\hline Credit to private sector & 23,090 & 23,294 & 25,011 & 29,449 & 33,962 \\
\hline Other items (net) & $-9,065$ & $-11,201$ & $-11,405$ & $-15,339$ & $-16,275$ \\
\hline Liabilities to the private sector & 36,381 & 37,653 & 41,221 & 49,439 & 54,572 \\
\hline Private capital and reserves & 6,869 & 8,097 & 9,269 & 11,340 & 11,672 \\
\hline Currency outside banks & 1,373 & 1,502 & 1,709 & 1,957 & 2,425 \\
\hline Deposit liabilities & 22,437 & 22,173 & 22,754 & 27,513 & 31,311 \\
\hline \multicolumn{6}{|l|}{ Of which } \\
\hline Foreign currency & 5,385 & 6,219 & 6,151 & 7,180 & 9,452 \\
\hline Fund-raising instruments 2/ & 4,920 & 1,493 & 1,272 & 2,045 & 2,129 \\
\hline \multirow[t]{2}{*}{ Other liabilities } & 781 & 4,389 & 6,217 & 6,584 & 7,036 \\
\hline & \multicolumn{5}{|c|}{ (Annual percentage change in relation to previous year's liabilities to the private sector) } \\
\hline Net international reserves & 5.0 & 1.3 & 3.4 & 19.1 & 21.7 \\
\hline Net domestic assets & 0.6 & -3.6 & 0.0 & -11.4 & -5.8 \\
\hline \multicolumn{6}{|l|}{ Of which } \\
\hline Credit to the public sector & -5.3 & 1.7 & -4.0 & -12.6 & -13.1 \\
\hline Credit to the private sector & 6.0 & 0.6 & 4.6 & 10.8 & 9.1 \\
\hline \multirow[t]{2}{*}{ Liabilities to the private sector } & 9.8 & 3.5 & 9.5 & 19.9 & 10.4 \\
\hline & \multicolumn{5}{|c|}{ (Annual percentage changes) } \\
\hline Net domestic assets & 1.8 & -11.1 & 0.0 & -44.6 & -49.2 \\
\hline Credit to the private sector & 9.4 & 0.9 & 7.4 & 17.7 & 15.3 \\
\hline Liabilities of the private sector & 9.8 & 3.5 & 9.5 & 19.9 & 10.4 \\
\hline Private capital and reserves & 13.7 & 17.9 & 14.5 & 22.3 & 2.9 \\
\hline Deposits & 13.4 & -1.2 & 2.6 & 20.9 & 13.8 \\
\hline
\end{tabular}

Source: Central Bank of Trinidad and Tobago.

$1 /$ This consolidates the central bank, commercial banks, trust and mortgage companies, and finance houses and merchant banks. 2/ These are guaranteed investments backed by government securities or mortagages, e.g., investment note certificates, secured commercial paper, floating rate tax-free debentures and mortgage pass-through securities. 
Table 18. Trinidad and Tobago: Monetary Survey

(In millions of Trinidad and Tobago dollars)

\begin{tabular}{|c|c|c|c|c|c|}
\hline & 2001 & 2002 & 2003 & 2004 & 2005 \\
\hline Net foreign assets & 12,341 & 12,856 & 14,065 & 21,901 & 32,808 \\
\hline Net international reserves & 11,832 & 12,317 & 13,584 & 21,459 & 32,174 \\
\hline Monetary authorities & 11,631 & 11,978 & 13,834 & 18,487 & 29,813 \\
\hline Commercial banks & 201 & 339 & -250 & 2,972 & 2,361 \\
\hline Other foreign assets/liabilities & 509 & 539 & 481 & 442 & 634 \\
\hline Monetary authorities & 509 & 539 & 481 & 442 & 634 \\
\hline Commercial banks & 0 & 0 & 0 & 0 & 0 \\
\hline Net domestic assets & 3,488 & 2,895 & 1,536 & $-3,463$ & $-6,459$ \\
\hline Domestic credit & 13,840 & 15,037 & 14,458 & 13,188 & 11,475 \\
\hline Net credit to public sector & $-3,261$ & $-2,825$ & $-4,925$ & $-10,243$ & $-16,295$ \\
\hline Net credit to government & $-3,427$ & $-3,797$ & $-5,041$ & $-10,115$ & $-16,939$ \\
\hline Credit to government & 2,796 & 2,782 & 3,330 & 3,416 & 4,037 \\
\hline Loans & 2,796 & 2,782 & 3,330 & 3,416 & 4,037 \\
\hline Advances & 4 & 3 & 9 & 0 & 7 \\
\hline T-bill holdings & 1,241 & 1,406 & 1,117 & 1,828 & 1,883 \\
\hline Other securities & 1,551 & 1,373 & 2,203 & 1,588 & 2,147 \\
\hline Other & 0 & 0 & 0 & 0 & 0 \\
\hline Liabilities & 6,223 & 6,579 & 8,371 & 13,531 & 20,976 \\
\hline Deposits of government & 6,223 & 6,579 & 8,371 & 13,531 & 20,976 \\
\hline \multicolumn{6}{|l|}{ Of which } \\
\hline Oil revenue stabilization fund & 1,015 & 1,015 & 1,567 & 2,846 & 5,485 \\
\hline Net credit to other public sector (nonfinancial & 166 & 972 & 116 & -129 & 644 \\
\hline Credit to other public sector (nonfinancial) & 1,794 & 2,263 & 1,599 & 1,701 & 3,259 \\
\hline Loans & 1,794 & 2,263 & 1,599 & 1,701 & 3,259 \\
\hline Advances & 1,086 & 1,225 & 1,246 & 1,575 & 2,883 \\
\hline Securities & 708 & 1,038 & 353 & 126 & 376 \\
\hline Other & 0 & 0 & 0 & 0 & 0 \\
\hline Liabilities & 1,628 & 1,291 & 1,483 & 1,830 & 2,615 \\
\hline Deposits of other public sector & 1,628 & 1,291 & 1,483 & 1,830 & 2,615 \\
\hline Other & 0 & 0 & 0 & 0 & 0 \\
\hline Credit to the economy & 17,101 & 17,862 & 19,383 & 23,431 & 27,770 \\
\hline Nonbank financial institutions & 3,056 & 3,171 & 4,149 & 4,284 & 3,108 \\
\hline Credit to the private sector & 14,045 & 14,691 & 15,234 & 19,147 & 24,662 \\
\hline Enterprises & 7,783 & 8,248 & 8,799 & 10,926 & 13,026 \\
\hline Households & 6,262 & 6,442 & 6,435 & 8,221 & 11,636 \\
\hline Other items (net) & $-10,351$ & $-12,142$ & $-12,923$ & $-16,651$ & $-17,934$ \\
\hline Liabilities to the private sector & 25,327 & 26,518 & 27,501 & 32,746 & 41,030 \\
\hline Private capital and reserves & 4,749 & 5,383 & 5,951 & 7,154 & 7,341 \\
\hline Broad money & 20,578 & 21,135 & 21,551 & 25,592 & 33,689 \\
\hline Money and quasi-money & 19,239 & 19,802 & 20,415 & 23,792 & 28,943 \\
\hline Currency outside banks & 1,373 & 1,502 & 1,709 & 1,957 & 2,425 \\
\hline Deposits & 17,865 & 18,300 & 18,706 & 21,834 & 26,518 \\
\hline \multicolumn{6}{|l|}{ Of which } \\
\hline Foreign currency & 5,385 & 6,219 & 6,151 & 7,180 & 9,452 \\
\hline Fund-raising instruments & 1,340 & 1,333 & 1,136 & 1,800 & 1,674 \\
\hline
\end{tabular}

Source: Central Bank of Trinidad and Tobago. 
Table 19. Trinidad and Tobago: Summary Accounts of the Central Bank (In millions of Trinidad and Tobago dollars)

\begin{tabular}{|c|c|c|c|c|c|}
\hline & 2001 & 2002 & 2003 & 2004 & 2005 \\
\hline Net foreign assets & 12,140 & 12,517 & 14,315 & 18,929 & 30,447 \\
\hline Net international reserves & 11,631 & 11,978 & 13,834 & 18,487 & 29,813 \\
\hline Assets & 11,741 & 12,079 & 13,935 & 18,589 & 29,915 \\
\hline Liabilities & 110 & 102 & 102 & 102 & 102 \\
\hline Other foreign assets/liabilities & 509 & 539 & 481 & 442 & 634 \\
\hline Net domestic assets & $-6,326$ & $-6,932$ & $-8,433$ & $-13,144$ & $-22,412$ \\
\hline Domestic credit & $-5,495$ & $-5,956$ & $-7,620$ & $-12,338$ & $-20,058$ \\
\hline Net credit to public sector & $-5,874$ & $-6,336$ & $-8,000$ & $-12,718$ & $-20,437$ \\
\hline Net credit to government & $-6,113$ & $-6,502$ & $-8,156$ & $-12,871$ & $-20,586$ \\
\hline Claims on central government & $-2,333$ & $-2,667$ & $-3,100$ & $-6,104$ & $-7,407$ \\
\hline Loans & 0 & 11 & 0 & 0 & 0 \\
\hline Less: sterilization (blocked account) & $-2,334$ & $-2,677$ & $-3,100$ & $-6,105$ & $-7,407$ \\
\hline $\begin{array}{l}\text { Deposits of central government } \\
\text { Of which }\end{array}$ & 3,780 & 3,836 & 5,056 & 6,767 & 13,180 \\
\hline Oil revenue stabilization fund & 1,015 & 1,015 & 1,567 & 2,846 & 5,485 \\
\hline Net credit to rest of public sector & 239 & 167 & 156 & 154 & 149 \\
\hline Claims on the rest of public sector & 309 & 299 & 280 & 245 & 234 \\
\hline Deposits of rest of public sector & 70 & 133 & 124 & 92 & 85 \\
\hline Net claims on financial institutions & 380 & 380 & 380 & 380 & 380 \\
\hline Other items (net) & -832 & -976 & -813 & -806 & $-2,355$ \\
\hline Reserve money & 5,814 & 5,585 & 5,882 & 5,785 & 8,035 \\
\hline Currency issue & 1,843 & 2,005 & 2,295 & 2,554 & 2,991 \\
\hline Deposits of commercial banks & 3,466 & 3,072 & 2,955 & 2,783 & 4,673 \\
\hline Deposits of nonbank financial institutions & 505 & 509 & 632 & 449 & 371 \\
\hline
\end{tabular}

Source: Central Bank of Trinidad and Tobago. 
Table 20. Trinidad and Tobago: Consolidated Accounts of the Commercial Banks (In millions of Trinidad and Tobago dollars)

\begin{tabular}{|c|c|c|c|c|c|}
\hline & 2001 & 2002 & 2003 & 2004 & 2005 \\
\hline Net foreign assets & 201 & 339 & -250 & 2,972 & 2,361 \\
\hline Net international reserves & 201 & 339 & -250 & 2,972 & 2,361 \\
\hline Other foreign assets/liabilities & 0 & 0 & 0 & 0 & 0 \\
\hline Monetary reserves and currency holdings & 3,936 & 3,535 & 3,541 & 3,353 & 4,758 \\
\hline Deposits with central bank & 3,466 & 3,032 & 2,955 & 2,756 & 4,192 \\
\hline Local currency holdings & 470 & 503 & 586 & 597 & 566 \\
\hline Net domestic assets & 10,701 & 10,756 & 10,983 & 10,537 & 14,113 \\
\hline Domestic credit & 19,209 & 20,864 & 21,826 & 25,456 & 31,485 \\
\hline Net credit to public sector & 2,612 & 3,510 & 3,075 & 2,474 & 4,142 \\
\hline Net credit to government & 2,686 & 2,706 & 3,115 & 2,757 & 3,647 \\
\hline Credit to government & 2,796 & 2,772 & 3,329 & 3,416 & 4,037 \\
\hline Loans & 1,245 & 1,398 & 1,126 & 1,828 & 1,890 \\
\hline Advances & 4 & 3 & 9 & 0 & 7 \\
\hline Treasury bill holdings & 1,241 & 1,395 & 1,117 & 1,828 & 1,883 \\
\hline Other securities & 1,551 & 1,373 & 2,203 & 1,588 & 2,146 \\
\hline Liabilities & 109 & 66 & 215 & 659 & 390 \\
\hline Deposits of government & 109 & 66 & 215 & 659 & 390 \\
\hline Net credit to other public sector (nonfinancial) & -74 & 805 & -40 & -283 & 495 \\
\hline Credit to other public sector (nonfinancial) & 1,484 & 1,964 & 1,319 & 1,456 & 3,025 \\
\hline Loans & 1,484 & 1,964 & 1,319 & 1,456 & 3,025 \\
\hline Advances & 777 & 925 & 966 & 1,330 & 2,649 \\
\hline Securities & 708 & 1,038 & 353 & 126 & 376 \\
\hline Liabilities & 1,558 & 1,159 & 1,359 & 1,738 & 2,530 \\
\hline Deposits of other public sector & 1,558 & 1,159 & 1,359 & 1,738 & 2,530 \\
\hline Claims on rest of the economy & 16,596 & 17,353 & 18,752 & 22,983 & 27,343 \\
\hline Nonbank financial institutions & 2,551 & 2,663 & 3,518 & 3,836 & 2,681 \\
\hline Private sector & 14,045 & 14,691 & 15,234 & 19,147 & 24,662 \\
\hline Enterprises & 7,783 & 8,248 & 8,799 & 10,926 & 13,026 \\
\hline Households & 6,262 & 6,442 & 6,435 & 8,221 & 11,636 \\
\hline Other items (net) & $-8,507$ & $-10,108$ & $-10,844$ & $-14,920$ & $-17,373$ \\
\hline Liabilities to the central bank & 382 & 380 & 382 & 381 & 380 \\
\hline Liabilities to the private sector & 22,614 & 23,683 & 24,656 & 28,989 & 33,859 \\
\hline Private capital and reserves & 4,749 & 5,383 & 5,951 & 7,154 & 7,341 \\
\hline Deposits & 17,865 & 18,300 & 18,706 & 21,834 & 26,518 \\
\hline \multicolumn{6}{|l|}{ Of which } \\
\hline Foreign currency & 5,385 & 6,219 & 6,151 & 7,180 & 9,452 \\
\hline Demand deposits & 4,238 & 5,393 & 5,595 & 6,866 & 9,069 \\
\hline Time deposits & 5,318 & 4,222 & 3,410 & 3,858 & 4,722 \\
\hline Savings deposits & 8,309 & 8,685 & 9,701 & 11,111 & 12,727 \\
\hline Fund raising instruments & 1,340 & 1,333 & 1,136 & 1,800 & 1,674 \\
\hline
\end{tabular}

Source: Central Bank of Trinidad and Tobago. 
Table 21. Trinidad and Tobago: Commercial Bank Loans and Advances

\begin{tabular}{|c|c|c|c|c|c|}
\hline & 2001 & 2002 & 2003 & 2004 & $2005 p$ \\
\hline & \multicolumn{5}{|c|}{ (In millions of Trinidad and Tobago dollars) } \\
\hline Total loans and advances & 14,750 & 14,731 & 16,581 & 21,117 & 28,734 \\
\hline Public sector & 1,235 & 985 & 1,029 & 1,570 & 3,168 \\
\hline Central government & 33 & 23 & 21 & 4 & 7 \\
\hline Local governments & 66 & 19 & 1 & 6 & 4 \\
\hline Public financial institutions & 420 & 33 & 33 & 116 & 369 \\
\hline Statutory boards & 102 & 357 & 574 & 1,002 & 1,113 \\
\hline Public enterprises & 615 & 553 & 401 & 442 & 1,675 \\
\hline Private sector & 13,515 & 13,746 & 15,551 & 19,546 & 25,566 \\
\hline Businesses & 7,234 & 7,287 & 9,097 & 11,216 & 13,769 \\
\hline \multicolumn{6}{|l|}{ Of which: } \\
\hline Agriculture & 124 & 80 & 106 & 116 & 92 \\
\hline Petroleum & 263 & 255 & 449 & 403 & 499 \\
\hline Manufacturing & 1,470 & 2,073 & 1,725 & 1,727 & 1,925 \\
\hline Construction & 551 & 702 & 1,140 & 1,198 & 1,195 \\
\hline Real estate & 97 & 106 & 42 & 809 & 1,232 \\
\hline Services & 4,730 & 4,071 & 5,635 & 6,963 & 8,825 \\
\hline \multicolumn{6}{|l|}{ Of which } \\
\hline Distribution & 1,086 & 954 & 1,226 & 1,301 & 2,068 \\
\hline Financial services & 1,774 & 1,771 & 2,866 & 3,485 & 4,386 \\
\hline Financial institutions & 198 & 248 & 1,170 & 955 & 1,123 \\
\hline Consumers & 6,281 & 6,459 & 6,455 & 8,331 & 11,796 \\
\hline & \multicolumn{5}{|c|}{ (In percent of loans and advances) } \\
\hline Public sector & 8.4 & 6.7 & 6.2 & 7.4 & 11.0 \\
\hline Private sector & 0.0 & 0.0 & 0.0 & 0.0 & 89.0 \\
\hline Businesses & 49.0 & 49.5 & 54.9 & 53.1 & 47.9 \\
\hline \multicolumn{6}{|l|}{ Of which: } \\
\hline Agriculture & 0.8 & 0.5 & 0.6 & 0.5 & 0.3 \\
\hline Petroleum & 1.8 & 1.7 & 2.7 & 1.9 & 1.7 \\
\hline Manufacturing & 10.0 & 14.1 & 10.4 & 8.2 & 6.7 \\
\hline Construction & 3.7 & 4.8 & 6.9 & 5.7 & 4.2 \\
\hline Real estate & 0.7 & 0.7 & 0.3 & 3.8 & 4.3 \\
\hline Services & 32.1 & 27.6 & 34.0 & 33.0 & 30.7 \\
\hline \multicolumn{6}{|l|}{ Of which } \\
\hline Distribution & 7.4 & 6.5 & 7.4 & 6.2 & 7.2 \\
\hline Financial services & 12.0 & 12.0 & 17.3 & 16.5 & 15.3 \\
\hline Financial institutions & 1.3 & 1.7 & 7.1 & 4.5 & 3.9 \\
\hline Consumers & 42.6 & 43.8 & 38.9 & 39.5 & 41.1 \\
\hline
\end{tabular}

Source: Central Bank of Trinidad and Tobago. 
Table 22. Trinidad and Tobago: Summary Accounts of the Nonbank Financial Institutions (NBFIs)

(In millions of Trinidad and Tobago dollars)

\begin{tabular}{|c|c|c|c|c|c|}
\hline & 2001 & 2002 & 2003 & 2004 & 2005 \\
\hline & \multicolumn{4}{|c|}{ I. Trust and Mortgage Companies } & \\
\hline Monetary reserves and currency & 350 & 351 & 433 & 429 & 425 \\
\hline Net domestic assets & 6,291 & 6,047 & $\mathbf{7 , 4 4 8}$ & 7,389 & 7,309 \\
\hline Credit to public sector & 894 & 916 & 1,583 & 1,725 & 1,500 \\
\hline Central government & 555 & 548 & 616 & 462 & 625 \\
\hline Rest of public sector & 339 & 368 & 967 & 1,262 & 875 \\
\hline Local government & 0 & 0 & 15 & 22 & 22 \\
\hline Statutory bodies & 0 & -1 & -44 & 12 & 14 \\
\hline Public utilities & 304 & 302 & 314 & 366 & 311 \\
\hline Nonfinancial state enterprises & 35 & 67 & 682 & 863 & 529 \\
\hline Credit to private sector & 6,217 & 6,146 & 7,217 & 6,250 & 6,056 \\
\hline Credit to other financial instns (net) & -83 & 48 & -60 & 656 & 1,120 \\
\hline Credit to commercial banks (net) & 746 & 943 & 804 & 1,107 & 1,340 \\
\hline Credit to nonbank financial institutions & -561 & -780 & -788 & -476 & -232 \\
\hline Credit to public financial institutions & -268 & -115 & -77 & 25 & 12 \\
\hline Other items (net) & -736 & $-1,064$ & $-1,291$ & $-1,241$ & $-1,367$ \\
\hline Liabilities to the central bank & 5 & 4 & 3 & 2 & 1 \\
\hline Liabilities to the private sector & 7,960 & 8,197 & 10,098 & 9,813 & $\mathbf{9 , 8 7 5}$ \\
\hline Private capital and reserves & 1,324 & 1,802 & 2,220 & 1,996 & 2,142 \\
\hline Time deposits & 2,274 & 1,845 & 1,525 & 988 & 243 \\
\hline \multicolumn{6}{|l|}{ Of which } \\
\hline Foreign currency & 0 & 0 & 0 & 0 & 0 \\
\hline Other liabilities $1 /$ & 4,362 & 4,549 & 6,353 & 6,829 & 7,491 \\
\hline Of which & & & & & \\
\hline \multirow[t]{2}{*}{ Fund-raising instruments 1 / } & 3,581 & 160 & 136 & 245 & 455 \\
\hline & \multicolumn{4}{|c|}{ II. Finance Houses and Merchant Banks } & \\
\hline Net foreign assets & 534 & 751 & 1,370 & 3,186 & 3,110 \\
\hline Assets & 1,243 & 2,123 & 3,034 & 3,979 & 4,884 \\
\hline Liabilities & 709 & 1,372 & 1,663 & 792 & 1,774 \\
\hline Monetary reserves and currency & 162 & 155 & 194 & 197 & 190 \\
\hline Net domestic assets & 1,631 & 1,141 & 973 & 1,315 & -453 \\
\hline Credit to public sector & 231 & 382 & 303 & 266 & 85 \\
\hline Central government & 163 & 160 & 161 & 143 & 170 \\
\hline Rest of the public sector & 68 & 222 & 142 & 124 & -85 \\
\hline Local government & 0 & 0 & 0 & 0 & 0 \\
\hline Statutory bodies & 2 & 155 & 147 & 138 & 3 \\
\hline Public utilities & 113 & 27 & 12 & 12 & 12 \\
\hline Nonfinancial state enterprises & -47 & 39 & -18 & -26 & -99 \\
\hline Credit to private sector & 2,828 & 2,457 & 2,561 & 4,052 & 3,244 \\
\hline Credit to other financial institutions (net) & -14 & 13 & 74 & 779 & 0 \\
\hline Credit to private financial institutions (net) & 187 & 260 & 373 & 944 & 0 \\
\hline Credit to public financial institutions (net) & -201 & -248 & -299 & -165 & 0 \\
\hline Other items (net) & $-1,414$ & $-1,711$ & $-1,964$ & $-3,782$ & $-3,782$ \\
\hline Liabilities to the central bank & 30 & 20 & 14 & 8 & 8 \\
\hline Liabilities to the private sector & 3,094 & 2,939 & 3,622 & 6,880 & 7,003 \\
\hline Private capital and reserves & 797 & 911 & 1,099 & 2,190 & 2,453 \\
\hline Total deposits & 2,298 & 2,027 & 2,523 & 4,690 & 4,550 \\
\hline Of which & & & & & \\
\hline Foreign currency & 0 & 0 & 0 & 0 & 0 \\
\hline Time deposits & 2,298 & 2,027 & 2,523 & 4,690 & 4,550 \\
\hline
\end{tabular}

Source: Central Bank of Trinidad and Tobago.

1/ From June 2000, some instruments were reclassified as fund raising instruments. 
Table 23. Trinidad \& Tobago: Financial Soundness Indicators - Banking Sector

\begin{tabular}{|c|c|c|c|c|c|}
\hline & 2001 & 2002 & 2003 & 2004 & 2005 \\
\hline & \multicolumn{5}{|c|}{ (In percent, unless indicated otherwise) } \\
\hline \multicolumn{6}{|l|}{ Capital adequacy } \\
\hline Regulatory capital to risk-weighted assets & 19.5 & 20.6 & 20.3 & 19.3 & 18.2 \\
\hline Regulatory Tier I capital to risk-weighted assets & 17.6 & 17.3 & 18.2 & 17.4 & 16.4 \\
\hline Regulatory Tier II capital-to-risk-weighted assets & 1.9 & 3.3 & 2.2 & 1.9 & 1.7 \\
\hline Regulatory capital-to-total assets & 11.1 & 11.7 & 12.4 & 13.2 & 11.6 \\
\hline \multicolumn{6}{|l|}{ Banking sector asset composition } \\
\hline \multicolumn{6}{|l|}{ Sectoral distribution of loans-to-total loans } \\
\hline $\begin{array}{l}\text { Households } \\
\text { of which: }\end{array}$ & 42.8 & 41.3 & 40.4 & 38.9 & 41.0 \\
\hline Proportion secured as mortgage loans & 10.2 & 11.4 & 11.3 & 18.3 & 27.2 \\
\hline Financial sector & 12.5 & 12.1 & 17.6 & 17.8 & 16.9 \\
\hline Oil and gas sector & 5.5 & 4.2 & 4.6 & 2.9 & 2.8 \\
\hline Construction & 3.8 & 4.6 & 7.4 & 6.4 & 5.9 \\
\hline Transport and communication & 4.9 & 3.7 & 3.7 & 3.6 & 2.7 \\
\hline Non-residents & 2.2 & 3.2 & 2.3 & 4.6 & 5.6 \\
\hline \multicolumn{6}{|l|}{ Geographic distribution of loans-to-total loans } \\
\hline Domestic & 97.8 & 96.8 & 97.7 & 96.3 & 93.8 \\
\hline Foreign & 2.2 & 3.2 & 2.3 & 3.7 & 6.2 \\
\hline Large exposures-to-capital & --- & --- & 84.4 & 82.9 & $\mathrm{n} / \mathrm{a}$ \\
\hline Gross asset position in derivatives to capital & 6.7 & 5.8 & 8.3 & 24.2 & $\mathrm{n} / \mathrm{a}$ \\
\hline Gross liability position in derivatives to capital & 1.4 & 1.3 & 4.5 & 21.7 & $\mathrm{n} / \mathrm{a}$ \\
\hline Foreign currency loans-to-total loans & 30.1 & 31.2 & 17.2 & 25.6 & 23.9 \\
\hline \multicolumn{6}{|l|}{ Banking sector asset quality } \\
\hline Nonperforming loans-to-gross loans & 3.2 & 3.6 & 2.0 & 3.9 & 1.7 \\
\hline Nonperforming loans (net of provisions)-to-capital & 0.6 & -1.0 & -3.0 & 6.0 & 0.3 \\
\hline Specific provisions-to-impaired assets & 57.7 & 70.8 & 117.5 & 40.3 & 67.7 \\
\hline Specific provisions-to-gross lending & 1.9 & 2.6 & 2.3 & 1.6 & 1.1 \\
\hline \multicolumn{6}{|l|}{ Banking sector earnings and profitability } \\
\hline Return on equity & 17.2 & 20.0 & 16.9 & 27.5 & 20.2 \\
\hline Return on assets & 2.0 & 2.4 & 2.1 & 3.7 & 2.5 \\
\hline Interest margin-to-gross income & 36.5 & 43.0 & 47.9 & 38.3 & 41.0 \\
\hline Non-interest expenses-to-gross income & 39.5 & 43.3 & 50.7 & 41.3 & 43.6 \\
\hline Spread between average lending and deposit rates & 8.8 & 9.1 & 8.7 & 7.4 & 7.1 \\
\hline \multicolumn{6}{|l|}{ Banking sector liquidity } \\
\hline Liquid assets-to-total assets & 17.8 & 16.1 & 15.9 & 14.8 & 15.8 \\
\hline Liquid assets-to-total short-term liabilities & 24.8 & 22.6 & 22.3 & 20.5 & 21.7 \\
\hline Customer deposits-to-total (non-interbank) loans & 145.3 & 147.2 & 142.3 & 128.3 & 119.3 \\
\hline Foreign currency liabilities-to-total liabilities & 32.6 & 34.3 & 34.4 & 37.8 & 35.8 \\
\hline \multicolumn{6}{|l|}{ Banking sector sensitivity to market risk } \\
\hline Net open positions in FX-to-capital & -11.8 & -18.2 & -36.8 & -0.9 & -13.9 \\
\hline
\end{tabular}

Source: Central Bank of Trinidad and Tobago. 
Table 24. Trinidad and Tobago: Interest Rates 1/ (In percent per annum)

\begin{tabular}{|c|c|c|c|c|c|}
\hline & 2001 & 2002 & 2003 & 2004 & 2005 \\
\hline \multicolumn{6}{|l|}{ Commercial banks } \\
\hline \multicolumn{6}{|l|}{ Savings deposits } \\
\hline Ordinary & 3.0 & 2.3 & 2.0 & 1.8 & 1.5 \\
\hline Special & 5.3 & 3.1 & 3.0 & 2.5 & 2.4 \\
\hline \multicolumn{6}{|l|}{ Time deposits } \\
\hline 0-3 months & 6.6 & 3.5 & 3.1 & 2.7 & 2.7 \\
\hline $3-6$ months & 6.8 & 3.6 & 3.5 & 3.3 & 3.1 \\
\hline 6 months -1 year & 7.8 & 4.4 & 4.2 & 3.6 & 3.5 \\
\hline Deposits in U.S. dollars 2/ & 7.0 & 3.9 & 2.6 & 2.3 & 3.0 \\
\hline Basic prime rate & 15.0 & 12.0 & 11.5 & 9.5 & 9.0 \\
\hline Term & 15.3 & 13.6 & 11.3 & 9.5 & 9.1 \\
\hline Demand & 15.0 & 13.0 & 11.5 & 9.5 & 9.0 \\
\hline Overdraft & 15.5 & 13.6 & 11.5 & 9.5 & 9.1 \\
\hline Real estate mortgage & 16.0 & 11.8 & 12.5 & 9.5 & 9.3 \\
\hline Spread, 1-year time deposit to U.S. dollar rate & 0.8 & 0.5 & 1.6 & 1.3 & 0.5 \\
\hline Weighted average deposit rate & 5.7 & 3.7 & 2.5 & 2.3 & 1.8 \\
\hline Weighted average lending rate & 14.5 & 12.8 & 11.2 & 10.1 & 9.1 \\
\hline Spread, lending rate to deposit rate & 8.8 & 9.1 & 8.7 & 7.9 & 7.2 \\
\hline \multicolumn{6}{|l|}{ Thrift institutions } \\
\hline Savings deposits & 5.0 & 5.0 & 4.0 & 5.0 & 3.5 \\
\hline Time deposits ( $1-3$ years) & 8.0 & 8.0 & 6.0 & 8.0 & 5.8 \\
\hline Mortgage loans, residential & 13.3 & 12.5 & 12.5 & 12.0 & 9.5 \\
\hline \multicolumn{6}{|l|}{ Trust and mortgage finance companies } \\
\hline \multicolumn{6}{|l|}{ Time deposits } \\
\hline $1-3$ years & 9.7 & 9.8 & 9.8 & 4.6 & 4.7 \\
\hline \multicolumn{6}{|l|}{ Mortgage loans } \\
\hline Residential & 13.3 & 12.8 & 12.0 & 10.1 & 12.1 \\
\hline Commercial & 14.3 & 13.1 & 12.4 & 10.8 & 12.1 \\
\hline \multicolumn{6}{|l|}{ Finance houses and merchant banks } \\
\hline Time deposits & 9.8 & 8.0 & 7.6 & 8.3 & 6.1 \\
\hline Installment loans & 11.3 & 11.4 & 9.6 & 9.4 & 10.8 \\
\hline \multicolumn{6}{|l|}{ Nonbank financial institutions } \\
\hline Weighted average deposit rate & 10.1 & 7.7 & 6.8 & 5.8 & 5.6 \\
\hline Weighted average lending rate & 11.6 & 11.0 & 9.4 & 9.3 & 8.7 \\
\hline Spread lending rate to deposit rate & 1.5 & 3.3 & 2.6 & 3.5 & 3.0 \\
\hline Central bank rate & 13.0 & 7.3 & 7.0 & 7.0 & 8.0 \\
\hline Treasury bills 3 / & 8.3 & 4.8 & 4.8 & 4.8 & 4.8 \\
\hline
\end{tabular}

Sources: Central Bank of Trinidad and Tobago; and International Financial Statistics (IMF).

$1 /$ Median rates, unless otherwise specified.

2/ Weighted average deposit rate.

$3 /$ Weighted average discount rate for the year. 
Table 25. Trinidad and Tobago: Summary Balance of Payments

\begin{tabular}{|c|c|c|c|c|c|}
\hline & 2001 & 2002 & 2003 & 2004 & $\begin{array}{l}\text { Est. } \\
2005\end{array}$ \\
\hline & \multicolumn{5}{|c|}{ (In millions of U.S. dollars; unless otherwise indicated) } \\
\hline Current account balance & 449 & 76 & 961 & 1,803 & 3,964 \\
\hline Trade balance & 722 & 237 & 1,293 & 1,524 & 3,939 \\
\hline Exports & 4,304 & 3,920 & 5,205 & 6,403 & 9,664 \\
\hline Petroleum crude and refined & 1,735 & 1,768 & 2,386 & 1,643 & 3,038 \\
\hline Gas & 828 & 459 & 951 & 1,978 & 2,632 \\
\hline Petrochemicals & 823 & 647 & 907 & 1,522 & 1,922 \\
\hline Other & 918 & 1,046 & 962 & 1,260 & 2,072 \\
\hline Imports & 3,583 & 3,683 & 3,912 & 4,879 & 5,725 \\
\hline Fuel imports & 926 & 1,019 & 1,064 & 1,181 & 1,992 \\
\hline Capital & 1,048 & 1,165 & 1,053 & 1,747 & 1,125 \\
\hline Other & 1,608 & 1,499 & 1,794 & 1,951 & 2,608 \\
\hline Services and transfers (net) & -273 & -161 & -332 & 279 & 25 \\
\hline Nonfactor services (net) & 233 & 264 & 314 & 671 & 527 \\
\hline Factor income (net) & -539 & -480 & -705 & -446 & -554 \\
\hline Current transfers (net) & 33 & 55 & 59 & 54 & 53 \\
\hline Capital and financial account (net) $1 /$ & 22 & -27 & -627 & $-1,068$ & $-2,169$ \\
\hline Official, medium and long-term (net) & -49 & -51 & -74 & -230 & -45 \\
\hline Disbursements & 27 & 18 & 16 & 25 & 59 \\
\hline Amortizations & -76 & -69 & -90 & -255 & -104 \\
\hline Direct Investment (net) & 777 & 684 & 583 & 973 & 599 \\
\hline Inward & 835 & 791 & 808 & 1,001 & 940 \\
\hline Outward & 58 & 106 & 225 & 29 & 341 \\
\hline Commercial banks (net) & 199 & -22 & 94 & -524 & 97 \\
\hline Other private sector capital (net) $1 /$ & -904 & -639 & $-1,230$ & $-1,286$ & $-2,820$ \\
\hline Of which: net errors and omissions & -405 & -356 & -121 & -214 & -693 \\
\hline Overall balance & 470 & 49 & 334 & 735 & 1,795 \\
\hline Change in gross official reserves (increase -) & -470 & -49 & -334 & -735 & $-1,795$ \\
\hline \multicolumn{6}{|l|}{ Memorandum items: } \\
\hline & \multicolumn{4}{|c|}{ (In percent of GDP; unless otherwise specified) } & \\
\hline Current balance & 4.7 & 0.8 & 8.0 & 13.3 & 24 \\
\hline Exports & 45.3 & 40.4 & 43.3 & 47.3 & 60 \\
\hline Imports & 37.7 & 37.9 & 32.5 & 36.1 & 35 \\
\hline Gross international reserves (millions of US\$, end of period) & 1,876 & 1,924 & 2,258 & 2,993 & 4,787 \\
\hline Oil prices (WEO, spot crude) & 24.3 & 25.0 & 28.9 & 37.8 & 53 \\
\hline
\end{tabular}

Sources: Central Bank of Trinidad and Tobago, Central Statistical Office; and Fund staff estimates. 
Table 26. Trinidad and Tobago: Summary of Exports, f.o.b.

(In millions of U.S. dollars; unless otherwise indicated)

\begin{tabular}{|c|c|c|c|c|c|}
\hline & 2001 & 2002 & 2003 & 2004 & 2005 \\
\hline Total exports & $4,304.2$ & $3,920.0$ & $5,204.9$ & $6,402.9$ & 9663.9 \\
\hline Re-exports & 57.1 & 40.8 & 114.6 & 166.6 & $\ldots$ \\
\hline Domestic exports & $3,729.0$ & $3,604.2$ & $5,090.3$ & $6,236.3$ & $\ldots$ \\
\hline \multicolumn{5}{|l|}{ Crude petroleum } & $\ldots$ \\
\hline Volume (million barrels) & 18.3 & 24.8 & 26.0 & 20.5 & $\ldots$ \\
\hline Value & 453.0 & 586.0 & 814.9 & 821.6 & $\ldots$ \\
\hline Unit value & 24.8 & 23.6 & 31.3 & 40.1 & $\ldots$ \\
\hline \multicolumn{6}{|l|}{ Refined petroleum products } \\
\hline Volume (million barrels) & 54.3 & 53.4 & 52.7 & 40.3 & $\ldots$ \\
\hline Value & $1,282.3$ & $1,182.0$ & $1,571.1$ & 821.4 & $\ldots$ \\
\hline Unit value & 23.6 & 22.1 & 29.8 & 20.4 & $\ldots$ \\
\hline \multicolumn{6}{|l|}{ Liquified natural gas } \\
\hline Volume (million tonnes) & 2.8 & 4.1 & 8.9 & 9.4 & \\
\hline Volume (million btu) & 155.9 & 228.3 & 495.5 & 523.4 & $\ldots$ \\
\hline Value & 510.0 & 274.9 & 676.3 & $1,750.7$ & $\ldots$ \\
\hline Unit value & 3.3 & 1.2 & 1.4 & 3.3 & $\ldots$ \\
\hline \multicolumn{6}{|l|}{ Natural gas liquids } \\
\hline Volume (million barrels) & 7.7 & 8.8 & 10.2 & 10.1 & $\ldots$ \\
\hline Value & 310.0 & 184.3 & 274.4 & 227.3 & $\ldots$ \\
\hline Unit value & 40.3 & 20.9 & 26.9 & 22.5 & $\ldots$ \\
\hline \multirow{2}{*}{\multicolumn{6}{|c|}{ Anhydrous ammonia }} \\
\hline & & & & & \\
\hline Volume (thousand metric tons) & $3,217.0$ & $3,565.3$ & $3,974.0$ & $4,344.0$ & $\ldots$ \\
\hline Value & 435.0 & 338.0 & 490.0 & 919.0 & $\ldots$ \\
\hline Unit value & 135.2 & 94.8 & 123.3 & 211.6 & $\ldots$ \\
\hline \multicolumn{6}{|l|}{ Urea } \\
\hline Volume (thousand metric tons) & 515.0 & 674.8 & 648.0 & 575.0 & $\ldots$ \\
\hline Value & 65.0 & 63.0 & 101.1 & 102.7 & $\ldots$ \\
\hline Unit value & 126.2 & 93.4 & 156.0 & 178.6 & $\ldots$ \\
\hline \multicolumn{6}{|l|}{ Methanol } \\
\hline Volume (thousand metric tons) & $1,495.0$ & $2,829.0$ & $2,832.0$ & $3,344.0$ & $\ldots$ \\
\hline Value & 257.0 & 192.0 & 257.0 & 441.5 & $\ldots$ \\
\hline Unit value & 171.9 & 67.9 & 90.7 & 132.0 & $\ldots$ \\
\hline Other chemicals & 66.0 & 54.1 & 58.5 & 58.8 & $\ldots$ \\
\hline $\begin{array}{l}\text { Other products } \\
\text { Steel products }\end{array}$ & 868.8 & $1,004.9$ & 847.0 & $1,136.2$ & $\ldots$ \\
\hline Volume (thousand metric tons) & $1,939.0$ & $2,066.0$ & $1,903.0$ & $1,907.0$ & $\ldots$ \\
\hline Value & 286.0 & 332.0 & 321.1 & 412.1 & $\ldots$ \\
\hline Unit value & 147.5 & 160.7 & 168.7 & 216.1 & $\ldots$ \\
\hline \multicolumn{6}{|l|}{ Sugar } \\
\hline Volume (thousand metric tons) & 61.0 & 37.0 & 34.4 & 43.8 & $\ldots$ \\
\hline Value & 26.0 & 24.8 & 24.1 & 20.4 & $\ldots$ \\
\hline Unit value & 0.4 & 0.7 & 0.7 & 0.5 & $\ldots$ \\
\hline Other & 556.8 & 648.1 & 501.8 & 703.7 & $\ldots$ \\
\hline Food and live animals (excluding sugar) & 145.0 & 144.6 & 125.2 & 141.0 & $\ldots$ \\
\hline Beverages and tobacco & 94.6 & 101.1 & 101.1 & 83.1 & $\ldots$ \\
\hline Crude materials & 9.1 & 9.4 & 11.7 & 15.6 & $\ldots$ \\
\hline Animal and vegetable fats & 5.0 & 5.9 & 6.0 & 7.3 & $\ldots$ \\
\hline Manufactures (excluding steel) & 123.9 & 232.7 & 122.0 & 259.7 & $\ldots$ \\
\hline Machinery & 75.2 & 93.9 & 72.1 & 129.7 & $\ldots$ \\
\hline Miscellaneous manufactured articles & 103.8 & 60.2 & 63.1 & 66.7 & $\ldots$ \\
\hline Other exports and errors & 0.2 & 0.3 & 0.6 & 0.6 & $\ldots$ \\
\hline \multicolumn{6}{|l|}{ Memorandum items: } \\
\hline $\begin{array}{l}\text { Fuels and chemicals exports } \\
\text { (percent of total domestic production) }\end{array}$ & $\begin{array}{r}3,378.3 \\
79.5\end{array}$ & $\begin{array}{r}2,874.3 \\
74.1\end{array}$ & $\begin{array}{r}4,243.3 \\
83.4\end{array}$ & $\begin{array}{r}5,143.0 \\
81.9\end{array}$ & $\begin{array}{l}\cdots \\
\ldots\end{array}$ \\
\hline Nonfuels & $1,691.8$ & $1,652.0$ & $1,753.6$ & $2,658.2$ & $\ldots$ \\
\hline Crude oil exports/production (in percent) & 44.1 & 51.9 & 51.9 & 43.6 & $\cdots$ \\
\hline
\end{tabular}

Sources: Central Bank of Trinidad and Tobago; Central Statistical Office; and Fund staff estimates. 
Table 27. Trinidad and Tobago: Summary of Imports

\begin{tabular}{|c|c|c|c|c|c|}
\hline & 2001 & 2002 & 2003 & 2004 & 2005 \\
\hline & \multicolumn{5}{|c|}{ (In millions of U.S. dollars) } \\
\hline Total imports & 3582.5 & 3682.6 & 3911.9 & 4879.3 & 5724.6 \\
\hline Imports for processing & 9.7 & 9.8 & 12.7 & 13.7 & 14.7 \\
\hline Consumer goods & 662.7 & 645.5 & 755.8 & 837.3 & 1063.7 \\
\hline Nondurables & 400.9 & 382.5 & 450.8 & 494.3 & 616.3 \\
\hline Food & 272.5 & 247.4 & 285.8 & 325.9 & 408.3 \\
\hline Other & 128.4 & 135.1 & 164.9 & 168.4 & 208.0 \\
\hline Durables & 261.8 & 263.0 & 305.0 & 342.9 & 447.4 \\
\hline Raw materials and intermediate goods & 1438.8 & 1561.4 & 1621.0 & 1890.1 & 2907.3 \\
\hline Fuels & 925.4 & 1018.3 & 1064.2 & 1181.1 & 1992.2 \\
\hline Construction materials & 140.5 & 133.7 & 177.7 & 248.2 & 233.4 \\
\hline Other & 372.9 & 409.3 & 379.1 & 460.8 & 681.7 \\
\hline Capital goods & 1048.4 & 1165.3 & 1053.4 & 1747.2 & 1124.8 \\
\hline Transport equipment & 185.3 & 353.6 & 178.6 & 352.8 & 156.2 \\
\hline Oil and mining machinery & 51.1 & 86.8 & 85.4 & 326.9 & 87.2 \\
\hline Other & 812.0 & 724.9 & 789.4 & 1067.4 & 881.4 \\
\hline \multirow[t]{2}{*}{ Other Commodities } & 432.6 & 310.4 & 481.7 & 404.7 & 628.8 \\
\hline & \multicolumn{5}{|c|}{ (Annual percent change) } \\
\hline Total imports & 8.0 & 2.8 & 6.2 & 24.7 & 17.3 \\
\hline Consumer goods & 21.6 & -2.6 & 17.1 & 10.8 & 27.0 \\
\hline Raw materials and intermediate goods & -4.6 & 8.5 & 3.8 & 16.6 & 53.8 \\
\hline \multirow[t]{2}{*}{ Capital goods } & 24.8 & 11.2 & -9.6 & 65.9 & -35.6 \\
\hline & \multicolumn{5}{|c|}{ (In percent of GDP) } \\
\hline Total imports & 40.7 & 41.1 & 36.4 & 39.9 & 39.9 \\
\hline Consumer goods & 7.5 & 7.2 & 7.0 & 6.8 & 7.4 \\
\hline Raw materials and intermediate goods & 16.3 & 17.4 & 15.1 & 15.5 & 20.2 \\
\hline Capital goods & 11.9 & 13.0 & 9.8 & 14.3 & 7.8 \\
\hline
\end{tabular}

Sources: Central Bank of Trinidad and Tobago; Central Statistical Office; and Fund staff estimates. 
Table 28. Trinidad and Tobago: Imports by Country of Origin

\begin{tabular}{|c|c|c|c|c|c|}
\hline & 2001 & 2002 & 2003 & 2004 & 2005 \\
\hline & \multicolumn{5}{|c|}{ (In millions of U.S. dollars) } \\
\hline Imports, c.i.f. & $3,582.5$ & $3,682.6$ & $3,911.9$ & $4,879.3$ & $5,724.6$ \\
\hline CARICOM Countries & 121.1 & 92.4 & 92.2 & 97.7 & 111.7 \\
\hline Barbados & 23.4 & 30.7 & 22.2 & 22.7 & 28.6 \\
\hline Guyana & 16.3 & 15.8 & 22.4 & 26.0 & 22.0 \\
\hline Jamaica & 20.1 & 17.4 & 16.8 & 14.2 & 14.8 \\
\hline Other CARICOM countries & 61.3 & 28.5 & 30.8 & 34.8 & 46.3 \\
\hline Latin America Free Trade & 849.9 & 739.8 & 824.9 & 859.4 & $1,633.8$ \\
\hline Venezuela & 438.1 & 399.2 & 264.5 & 153.5 & 345.5 \\
\hline Brazil & 200.4 & 210.2 & 352.5 & 511.1 & 775.5 \\
\hline Other & 211.6 & 130.4 & 207.9 & 169.2 & 512.8 \\
\hline Central American Common Market & 27.3 & 25.6 & 23.5 & 21.6 & 27.8 \\
\hline United States & $1,315.9$ & $1,236.5$ & $1,179.7$ & $1,662.2$ & $1,643.2$ \\
\hline Canada & 91.7 & 104.1 & 116.9 & 107.9 & 123.0 \\
\hline European Economic Community & 642.2 & 586.7 & 697.1 & $1,080.2$ & 820.0 \\
\hline European Free Trade Association & 25.6 & 40.3 & 40.7 & 37.6 & 68.0 \\
\hline \multirow[t]{2}{*}{ Other } & 508.8 & 857.2 & 936.9 & $1,012.7$ & $1,297.1$ \\
\hline & \multicolumn{5}{|c|}{ (In percent of total) } \\
\hline CARICOM countries & 3.4 & 2.5 & 2.4 & 2.0 & 2.0 \\
\hline Barbados & 0.7 & 0.8 & 0.6 & 0.5 & 0.5 \\
\hline Guyana & 0.5 & 0.4 & 0.6 & 0.5 & 0.4 \\
\hline Jamaica & 0.6 & 0.5 & 0.4 & 0.3 & 0.3 \\
\hline Other CARICOM countries & 1.7 & 0.8 & 0.8 & 0.7 & 0.8 \\
\hline Latin America Free Trade & 23.7 & 20.1 & 21.1 & 17.6 & 28.5 \\
\hline Venezuela & 12.2 & 10.8 & 6.8 & 3.1 & 6.0 \\
\hline Brazil & 5.6 & 5.7 & 9.0 & 10.5 & 13.5 \\
\hline Other & 5.9 & 3.5 & 5.3 & 3.5 & 9.0 \\
\hline Central American Common Market & 0.8 & 0.7 & 0.6 & 0.4 & 0.5 \\
\hline United States & 36.7 & 33.6 & 30.2 & 34.1 & 28.7 \\
\hline Canada & 2.6 & 2.8 & 3.0 & 2.2 & 2.1 \\
\hline European Economic Community & 17.9 & 15.9 & 17.8 & 22.1 & 14.3 \\
\hline European Free Trade Association & 0.7 & 1.1 & 1.0 & 0.8 & 1.2 \\
\hline Other & 14.2 & 23.3 & 23.9 & 20.8 & 22.7 \\
\hline
\end{tabular}

Sources: Central Statistical Office; Central Bank of Trinidad and Tobago; and Fund staff estimates. 
Table 29. Trinidad and Tobago: Exports by Country of Destination

\begin{tabular}{|c|c|c|c|c|c|}
\hline & 2001 & 2002 & 2003 & 2004 & 2005 \\
\hline & \multicolumn{5}{|c|}{ (In millions of U.S. dollars) } \\
\hline Exports, f.o.b. & $4,304.2$ & $3,920.0$ & $5,204.9$ & $6,402.9$ & $9,663.9$ \\
\hline CARICOM countries & $1,034.7$ & 770.8 & 959.0 & 814.6 & $2,044.0$ \\
\hline Barbados & 267.6 & 179.5 & 203.7 & 213.8 & 405.7 \\
\hline Guyana & 88.4 & 83.0 & 149.9 & 122.4 & 266.8 \\
\hline Jamaica & 353.7 & 294.2 & 353.6 & 245.6 & 717.7 \\
\hline Other CARICOM countries & 325.0 & 214.1 & 251.8 & 232.8 & 653.8 \\
\hline Latin America Free Trade & 134.4 & 109.5 & 183.4 & 241.2 & 379.6 \\
\hline Venezuela & 51.1 & 24.1 & 42.2 & 17.9 & 23.7 \\
\hline Brazil & 13.7 & 18.5 & 30.8 & 36.9 & 69.5 \\
\hline Other & 69.6 & 66.9 & 110.4 & 186.4 & 286.4 \\
\hline Central American Common Market & 161.9 & 114.8 & 138.3 & 45.1 & 76.2 \\
\hline United States & $1,779.1$ & $1,803.5$ & $2,785.2$ & $4,471.8$ & $5,568.1$ \\
\hline Canada & 98.6 & 92.4 & 95.4 & 83.8 & 104.4 \\
\hline European Economic Community & 230.3 & 259.4 & 214.9 & 223.2 & 202.4 \\
\hline European Free Trade Association & 8.1 & 5.1 & 2.3 & 0.6 & 5.2 \\
\hline \multirow[t]{2}{*}{ Other } & 857.1 & 764.5 & 826.4 & 522.6 & $1,284.0$ \\
\hline & \multicolumn{5}{|c|}{ (In percent of total) } \\
\hline CARICOM countries & 24.0 & 19.7 & 18.4 & 12.7 & 21.2 \\
\hline Barbados & 6.2 & 4.6 & 3.9 & 3.3 & 4.2 \\
\hline Guyana & 2.1 & 2.1 & 2.9 & 1.9 & 2.8 \\
\hline Jamaica & 8.2 & 7.5 & 6.8 & 3.8 & 7.4 \\
\hline Other CARICOM countries & 7.6 & 5.5 & 4.8 & 3.6 & 6.8 \\
\hline Latin America Free Trade & 3.1 & 2.8 & 3.5 & 3.8 & 3.9 \\
\hline Venezuela & 1.2 & 0.6 & 0.8 & 0.3 & 0.2 \\
\hline Brazil & 0.3 & 0.5 & 0.6 & 0.6 & 0.7 \\
\hline Other & 1.6 & 1.7 & 2.1 & 2.9 & 3.0 \\
\hline Central American Common Market & 3.8 & 2.9 & 2.7 & 0.7 & 0.8 \\
\hline United States & 41.3 & 46.0 & 53.5 & 69.8 & 57.6 \\
\hline Canada & 2.3 & 2.4 & 1.8 & 1.3 & 1.1 \\
\hline European Economic Community & 5.4 & 6.6 & 4.1 & 3.5 & 2.1 \\
\hline European Free Trade Association & 0.2 & 0.1 & 0.0 & 0.0 & 0.1 \\
\hline Other & 19.9 & 19.5 & 15.9 & 8.2 & 13.3 \\
\hline
\end{tabular}

Sources: Central Statistical Office; Central Bank of Trinidad and Tobago; and Fund staff estimates. 
Table 30. Trinidad and Tobago: Public Sector External Debt

\begin{tabular}{|c|c|c|c|c|c|}
\hline & 2001 & 2002 & 2003 & 2004 & $\begin{array}{l}\text { Prel. } \\
2005\end{array}$ \\
\hline & \multicolumn{4}{|c|}{ (In millions of U.S. dollars) } & \\
\hline \multicolumn{6}{|l|}{ Public sector external debt } \\
\hline Debt outstanding (end of period) & 1,666 & 1,549 & 1,553 & 1,361 & 1,327 \\
\hline Drawings & 53 & 43 & 87 & 34 & 58 \\
\hline Amortization due & 61 & 69 & 90 & 226 & 92 \\
\hline Debt service & 183 & 198 & 206 & 336 & 186 \\
\hline Interest payments & 121 & 130 & 117 & 110 & 94 \\
\hline Rescheduling & 0 & 0 & 0 & 0 & 0 \\
\hline Valuation adjustment & -6 & 9 & 7 & 0 & 0 \\
\hline \multicolumn{6}{|l|}{ Central government } \\
\hline Debt outstanding (end of period) $1 /$ & 1,517 & 1,500 & 1,502 & 1,310 & 1,276 \\
\hline Drawings & 53 & 43 & 87 & 34 & 58 \\
\hline Amortization due & 61 & 69 & 90 & 226 & 92 \\
\hline Debt service & 183 & 198 & 206 & 336 & 186 \\
\hline Interest payments & 121 & 130 & 117 & 110 & 94 \\
\hline Rescheduling & 0 & 0 & 0 & 0 & 0 \\
\hline Valuation adjustment & -6 & 8 & 6 & 0 & 0 \\
\hline \multicolumn{6}{|l|}{ Public enterprises } \\
\hline Debt outstanding end of period & 149 & 50 & 51 & 51 & 51 \\
\hline Drawings & 0 & 0 & 0 & 0 & 0 \\
\hline Amortization & 0 & 0 & 0 & 0 & 0 \\
\hline Debt service & 0 & 0 & 0 & 0 & 0 \\
\hline Interest payments & 0 & 0 & 0 & 0 & 0 \\
\hline Rescheduling & 0 & 0 & 0 & 0 & 0 \\
\hline Valuation adjustment & 0 & 1 & 1 & 0.3 & 0 \\
\hline \multicolumn{6}{|l|}{ Central bank } \\
\hline Debt outstanding end of period & 0 & 0 & 0 & 0 & 0 \\
\hline Drawings & 0 & 0 & 0 & 0 & 0 \\
\hline Amortization & 0 & 0 & 0 & 0 & 0 \\
\hline Valuation adjustment & 0 & 0 & 0 & 0 & 0 \\
\hline \multirow[t]{3}{*}{ Interest payments } & 0 & 0 & 0 & 0 & 0 \\
\hline & $\mathrm{i}$ & & & & \\
\hline & \multicolumn{5}{|c|}{ (In percent of total outstanding debt, unless otherwise stated) } \\
\hline \multicolumn{6}{|l|}{ By creditor } \\
\hline Bilateral agencies & 2.3 & 2.0 & 1.5 & 1.1 & 0.8 \\
\hline Multilateral agencies & 36.0 & 37.5 & 39.2 & 41.8 & 41.7 \\
\hline Other & 61.4 & 55.4 & 58.1 & 55.6 & 57.5 \\
\hline Of which: bonds & 46.3 & 50.5 & 48.6 & 44.8 & 56.6 \\
\hline \multicolumn{6}{|l|}{ By maturity } \\
\hline Short term & 0.0 & 0.0 & 0.0 & 0.0 & 0.0 \\
\hline Medium term & 32.7 & 32.6 & 33.7 & 32.5 & 29.0 \\
\hline Long term & 67.3 & 67.4 & 66.3 & 67.5 & 71.0 \\
\hline Debt in percent of GDP & 19.7 & 17.6 & 14.8 & 11.1 & 9.2 \\
\hline Debt service in percent of exports of goods and ser & 3.7 & 4.4 & 3.5 & 4.6 & 1.8 \\
\hline Interest payment in percent of exports of goods anc & 2.5 & 2.8 & 2.0 & 1.5 & 0.9 \\
\hline
\end{tabular}

Sources: Ministry of Finance; Central Bank of Trinidad and Tobago; and Fund staff estimates.

1/ Excludes a short-term U.S. dollar denominated bond of US\$150 million provided by resident financial institutions in 1998. 